\title{
Reliability of the local truncations for the random tensor models renormalization group flow
}

\author{
Vincent Lahoche ${ }^{1, *}$ and Dine Ousmane Samary $\oplus^{1,2, \dagger}$ \\ ${ }^{1}$ Commissariat l'Énergie Atomique (CEA, LIST), 8 Avenue de la Vauve, 91120 Palaiseau, France \\ ${ }^{2}$ International Chair in Mathematical Physics and Applications (ICMPA-UNESCO Chair), \\ University of Abomey-Calavi, 072B.P.50 Cotonou, Republic of Benin
}

(Received 8 June 2020; accepted 31 July 2020; published 3 September 2020)

\begin{abstract}
The standard nonperturbative approaches of renormalization group for tensor models are generally focused on a purely local potential approximation (i.e., involving only generalized traces and products of them) and are showed to strongly violate the modified Ward identities. This paper is a continuation of our recent contribution [Phys. Rev. D 101, 106015 (2020), intended to investigate the approximation schemes compatible with Ward identities and constraints between $2 n$-points observables in the large $N$ limit. We consider separately two different approximations: In the first one, we try to construct a local potential approximation from a slight modification of the Litim regulator, so that it remains optimal in the usual sense, and preserves the boundary conditions in deep UV and deep IR limits. In the second one, we introduce derivative couplings in the truncations and show that the compatibility with Ward identities implies strong relations between $\beta$ functions, allowing one to close the infinite hierarchy of flow equations in the nonbranching sector, up to a given order in the derivative expansion. Finally, using an exact relation between correlations functions in large $N$ limit, we show that strictly local truncations are insufficient to reach the exact value for the critical exponent, highlighting the role played by these strong relations between observables taking into account the behavior of the flow; and the role played by the multitrace operators, discussed in the two different approximation schemes. In both cases, we compare our conclusions to the results obtained in the literature and conclude that, at a given order, by taking into account the exact functional relations between observables like Ward identities in a systematic way, we can strongly improve the physical relevance of the approximation for an exact renormalization group equation.
\end{abstract}

DOI: 10.1103/PhysRevD.102.056002

\section{INTRODUCTION}

Random tensor models (RTMs) were initially introduced in the quantum gravity context at the beginning of the 1990s [1-6], as a natural extension of random matrix models (RMMs) used to quantize two-dimensional gravity [7-12]. The strong revival of interest since the last decade started in 2009 with the discovery of complex colored RTMs [13-20]. In contrast with their formers, colored RTMs admit a $1 / N$ expansion controlled by the so-called Gurau degree of the corresponding Feynman graphs, which plays the same role as the genus for RMMs. The Gurau degree is reduced to the genus in dimension two, and may be defined as the sum of the genera of jackets, which are

\footnotetext{
*vincent.lahoche@cea.fr

†dine.ousmanesamary@cipma.uac.bj
}

Published by the American Physical Society under the terms of the Creative Commons Attribution 4.0 International license. Further distribution of this work must maintain attribution to the author(s) and the published article's title, journal citation, and DOI. Funded by SCOAP ${ }^{3}$. ribbon subgraphs of tensor diagrams. Interestingly, the Gurau degree is not a topological invariant but allows one to properly construct the leading order graphs in the large $N$ limit, called melons [18], in the same way as the planar graphs for RMM. The melons, in dimension $d>2$, correspond to particular simplicial decomposition (that we abusively call "triangulation") of the $d$-dimensional topological sphere $\mathcal{S}_{d}$. Moreover, they admit a continuum limit, with the entropy exponent corresponding to a branched-polymer phase [21-23]. Another important step in the development of RTMs was the discovery of the relation between the existence of an internal index symmetry and the $1 / N$ expansion. Indeed, the uncolored version of the initial complex colored models was introduced in [24], where the authors highlight the connection between the existence of a $1 / N$ expansion and the global $\mathrm{U}(N)^{\times d}$ invariance of the classical action. This simple observation leads to an extension of the colored formalism, in the same universality class [25]. This connection between symmetry and power counting has been extended a lot, and some models based on $\mathrm{O}(N)^{\times d}$ invariance have been successfully considered, providing triangulation for 
nonorientable manifolds [26,27]. Some other groups have been considered in the last years as well; see [28-31] for recent reviews.

Despite their recent connections with Sachdev-YeKitaev (SYK) models, and condensed matter physics [32-41], RTMs remain essentially, at this day, a promising road for a viable quantum gravity formalism. RTMs arise at the intersection between many current strategies to quantize gravity. Among their inspirations, one has RMMs [7-11], group field theories (GFTs) [42], and loop quantum gravity $[43,44]$. A recent consequence of GFTs and TMs was the development of tensorial group field theories [45-48] see also [49-52], which improve standard GFTs with the tensorial recipe for the construction of their interactions. This was at the origin of some promising renormalization group investigations in the GFTs phase space [53-62], revealing possible phase transitions compatible with a current scenario about space-time emergence.

However, in themselves RTMs admit a rich phase structure. This has been revealed by some analytic investigations conducted in the hope to go beyond the melonic university class and to discover a new continuum limit, with spectral dimension closer to the one of our four-dimensional spacetime. To this end, the next-to-leading order (NLO) of the $1 / N$ expansion plays an essential role. As for matrix models, the double scaling limit for tensor models is based on the observations that NLO contributions become critical at the same point as the melonic contribution. This suggests that we investigate the large $N$ limit and continuum limit in a correlated manner such that we retain the graphs of an arbitrary Gurau degree. In the case of matrix models, which can be achieved by sending $N \rightarrow \infty$ and $g \rightarrow g_{c}$, the theory is such that the product $N\left|g-g_{c}\right|^{(2-\gamma) / 2}$ (where $\gamma$ is the so-called entropy exponent) remains constant. The same behavior has been achieved for RTMs, in contrast with matrix models such that the double scaling limit leads to a summable series for dimensions less than six. Moreover, the double scaling procedure can be iterated, a multicritical scenario providing ultimately at the critical point a continuum limit so far from the branched polymer phase of the melonic limit. This multiscaling scenario remains an attractive and open perspective for RTM. To this end, nonperturbative renormalization group theory has been envisaged as a promising and alternative way of investigation of this critical behavior, easier than the heavy mathematical machinery used to formally construct the multiscaling investigations.

Using renormalization group theory to understand critical properties of such a discrete model has been firstly considered in the past for matrix models $[9,10]$; see also [63-73] for recent results. This comes from the original idea that the double scaling limit may be understood as a special parametrization invariance from the long-distance physics along a relevant direction, in complete analogy with what happens for standard critical phenomena. In Refs. $[9,10]$, the authors constructed such a renormalization group using perturbation theory and showed the existence of a non-Gaussian fixed point with a relevant direction and a critical exponent in qualitative agreement with the analytic calculations of the double scaling limit. The nonperturbative investigations started in [74,75] and using Wetterich-Morris formalism [76,77], showed significant improvements concerning the perturbative analysis and provided a tractable formalism to explore discrete gravity, in particular RTMs.

The success of this formalism, for matrix models, is because the critical exponent for the relevant direction seems to converge toward the exact (analytic) value provided by the double scaling limit when the truncation is enlarged. This observation, however, depends crudely on the specific scheme used to compute the critical exponents. This dependence, as pointed out in Ref. [75], could reflect a pathology of the local truncations used to solve the exact renormalization group equations. Indeed, all the considered versions of the nonperturbative renormalization group used a suitable version of the local potential approximation; which of course completely discards the effects arising from the symmetry breaking due to the regulation. This observation is supported by the fact that the heuristic strategy consisting of keeping only tadpole diagrams provides the most spectacular convergence toward the exact result, and such a scheme discards strong disagreements with modified Ward identities. The reliability of the method may be checked only because we have the exact result; thus, the question is, can we be confident with the ability of a purely local approximation in the discovering of new multicritical points for tensor models? Formally, there is no additional difficulty to pass from random matrices to random tensors. The main difference between RMM and RTM in practice is the proliferation of the interactions, and therefore of the beta functions with the rank of the truncation. Dealing with this difficulty remains tractable for not so large truncations, and the first investigations, as for matrix models, provided encouraging results, (re)discovering the critical fixed point corresponding to the double-scaling limit; having a single relevant direction with a critical exponent is in qualitative agreement with the exact analytic value $\theta_{\text {exact }}=d-2$.

However, as for matrix models, the quantitative agreement depends on the prescription used to compute the critical exponents or the flow equations, and once again is assumed to be a consequence of local truncations. To be used with confidence for discovering multicritical points beyond the double scaling limit, the formalism must allow having control of the approximations, and this is the more important property if we do not have the support of exact analytic calculations to estimate how much the approximation remains physically relevant, or if the discovered critical points are not an artifact of a bad parametrization of the full phase space. Fortunately, some physical guides are allowing one to test the reliability of the results obtained in a given prescription without knowledge of the exact 
solution. The compatibility with constraints arising from symmetries is one of these guides. For random tensor models, the constraints arise essentially from the symmetry breaking due to the regulator, which modifies the Ward identities. Purely local potential approximations strongly violate these identities. This fact was first pointed out in [78,79], but the proposed heuristic recipe, taking into account tadpole diagrams to accommodate with Ward identities, cannot be used confidently to investigate larger regions of the phase space than the small vicinity of the Gaussian fixed point containing the double-scaling critical point.

A systematic analysis of the influence of Ward identities on the behavior of the renormalization group flow was started for matrix models in [80]. The authors observed that an approximation scheme solving simultaneously Ward identities and flow equations strongly improves the value of the critical exponent related to the relevant direction, without additional prescription. This is based on the elementary observation that Ward identities and flow equations play a very symmetric role for discrete gravity models $[78,79,81-84]$ see also $[85,86]$ in the case of sextic interactions and an Ising-like model. Indeed, the modified Ward identities arise from the symmetry breaking due to the regulator, but this breaking is itself required to construct the renormalization group (RG) flow. This is radically different from the situation for ordinary gauge theories, where the RG flow exists independently, due to the nontrivial propagator of the gauge fields, without relation to the symmetry breaking which may arise by introducing the regulator function [87-90]. For RMMs and RTMs, however, the propagator is trivial, and the symmetry breaking is required to distinguish between UV and IR degrees of freedom. The modification of Ward identities, therefore, is more than a nontrivial aspect of the theory; it is a consequence of the existence of the RG flow itself. With this respect, a violation of Ward identities has to be considered as a serious problem then for ordinary gauge theory. For gauge theory, Ward identities reflect the gauge symmetry, which is unrelated to the scale hierarchy, but it is the case for RMMs and RTMs. In a recent result, we analyze the flow equations which dictate how to move though scales and also the Ward identities which dictate how to move through momentum space [81], and they have been understood as two complementary relations of the same thing.

In [80], we proposed two different ways to deal with Ward identities violations and constructed two approximate solutions compatible with them. The first one was to enlarge the truncation with momentum-dependent interaction, reflecting the symmetry breaking. This procedure, as expected, strongly improves the value of the computed critical exponent for the relevant eigendirection at the critical point. However, the presence of derivative couplings, which have nonvanishing value at the fixed point, seems to introduce a spurious dependence on the regulator.
Such a dependence is, in fact, inevitable in any approximation scheme, and we expect that the sensibility for small deformation of the regulating function may be a good test for the quality of the approximation. From a simple deformation, we showed that the critical exponent does not change significantly around the Litim regulator, in agreement with the familiar claim about its efficiency. The second strategy was to consider a modified regulator, including fine-tuned counterterms. These counterterms do not change the UV and IR boundary conditions and are chosen to cancel the momentum-dependent terms in Ward identities using local potential approximation, such that the violation remains as small as possible in the considered range of couplings investigated by the RG flow (expecting that we remain not so far from the Gaussian fixed point, which is essentially the same assumption ensuring the validity of the truncation method). With this method, we found a fixed point, and a critical exponent in very strong agreement with the exact value, ensuring that, up to this fine adjustment of the regulator, the local approximation may be used in practice to solve both flow equations and Ward identities.

In this paper, we continue the same analysis for tensor models. We start with complex and real RTMs, having $\mathrm{U}(N)$ invariance, and in both cases, we construct two kinds of approximations, compatible with Ward identities, and investigate the continuum limit through the properties of the resulting fixed points. In detail, the outline is the following: In Sec. II, we recall some basics about RTMs, nonperturbative RG formalism, and Ward identities. We provide useful definitions and present the notations, as well as the elementary notions, as the proper notion of canonical dimension for RTMs. In Sec. III we build a local truncation scheme based on a progressive modification of the (Litim) regulator [91-94], constructed to cancel the derivative couplings arising from Ward identity, at the order fixed by the truncation. We consider generic melonic truncations up to order eight, and higher truncation in the nonbranching sector, and show that the result is systematically better than those obtained from local truncations without a modified regulator. We show moreover that the connected invariant is insufficient to reach the exact value for the critical exponent and only a local truncation involving the product of local invariants and in agreement with Ward identities allows one to converge toward the exact result. In the last subsection, we use the recent effective vertex expansion (EVE) $[79,81]$ to obtain the inductive bound $\theta_{\mathrm{op}}=d-1$ toward which converges the critical exponent for a local truncation of arbitrary order, involving only melonic connected pieces. This shows that, independently of the regularization scheme and in agreement with the previous observation, the ultralocal approximations do not allow one to reach the exact value $\left(\theta_{\text {exact }}=d-2\right)$. In Sec. IV we propose an optimization criterion based on the sensibility of the results under small variations of the regulator and show that 
physical solutions are systematically stable. In Sec. V, we compare our results with another approximation scheme, including derivative couplings in the truncation, and show that, order by order in the derivative expansion. Including these operators allows one to close the (local) infinite hierarchical system of our equation provided by the exact $\mathrm{RG}$ equation.

\section{RG FLOW FOR U(N) RTMS AND LOCAL TRUNCATIONS}

In this section we provide some basic material for tensor models and nonperturbative RG formalism. Moreover, we introduce some useful definitions and properties that will be used to construct approximate solutions of the RG equation in the next sections.

\section{A. Wetterich-Morris formalism}

In the Wetterich-Morris formalism, the central object is the effective averaged action $\Gamma_{k}$, which follows the first order flow equation $[76,77]$ :

$$
\frac{\partial}{\partial k} \Gamma_{k}=\operatorname{Tr}\left(\frac{1}{\Gamma_{k}^{(2)}+R_{k}}\right) \frac{\partial}{\partial k} R_{k}
$$

where $\Gamma_{k}^{(2)}+R_{k}$ is the inverse of the effective 2-point function. The formal trace "Tr" depends on the nature of the fields involved in the equation and, the regulator function $R_{k}$ is a scale-dependent mass, chosen such that the degrees of freedom with momentum (smaller than $k$ ) are frozen out and discarded from the path integral defining the partition function. For the complex tensor models, this $k$-dependence partition function is given by

$$
Z_{k}[J, \bar{J}]=\int d T d \bar{T} e^{-S(T, \bar{T})-\bar{T} R_{k} T+\bar{J} T+\bar{T} J},
$$

where

(i) $T, \bar{T}$ are respectively the complex tensor and its conjugate, which rank is $d$ and size $N, T=$ $\left\{T_{n_{1}, \ldots, n_{d}}\right\}, T_{n_{1}, \ldots, n_{d}} \in \mathbb{C}$;

(ii) the regulator $R_{k}$ is a diagonal $N^{d} \times N^{d}$ matrix, i.e., $\left(R_{k}\right)_{\vec{n} \vec{n}^{\prime}}=r_{k}(\vec{n}) \delta_{\vec{n} \vec{n}^{\prime}}$; with $\vec{n}:=\left(n_{1}, \ldots, n_{d}\right)$;

(iii) the shorthand notations $\bar{T} R_{k} T$ and $\bar{J} T$ are respectively $\quad \bar{T} R_{k} T:=\sum_{\vec{n} \vec{n}^{\prime}} \bar{T}_{\vec{n}}\left(R_{k}\right)_{\vec{n} \vec{n}^{\prime}} T_{\vec{n}^{\prime}}$ and $\bar{J} T:=$ $\sum_{\vec{n}} \bar{J}_{\vec{n}} T_{\vec{n}}$, and

(iv) the classical action $S(T, \bar{T})$ is a sum of the connected invariant with respect to $U(N)^{\times d}$ transformations.

Let us set $R_{k}=0$. In the viewpoint where the degrees of freedom could be integrated out to build the RG flow, a global unitary invariant theory strongly provides some difficulties, particularly on the degrees of freedom of the initial condition. In standard field theory, we start with UV degrees of freedom, i.e. degrees of freedom having high momenta. This is suitable due to the existence of a nontrivial propagator, which provides a different size for quantum fluctuations. For the tensor models with a trivial propagator, all the fluctuations have the same size and we have a canonical notion of UV and IR. The UV is described by the classical action $S(T, \bar{T})$ and the IR, when all the fluctuations are integrated out, by the effective action $\Gamma$ :

$$
\Gamma[M, \bar{M}]+\ln Z_{k=0}[J, \bar{J}]=\bar{J} M+\bar{M} J,
$$

the classical field $M$ being defined as

$$
M=\frac{\partial}{\partial \bar{J}} \ln Z_{k}[J, \bar{J}] .
$$

Breaking the unitary invariance, the regulator defines a preferred order to make the partial integrations of the degrees of freedom, and provides a path to link UV and IR limits. At the same time, breaking the unitary invariance modifies the Ward identities; see [95-97] and references therein. From the global translation invariance of the partition function (2) and considering an infinitesimal unitary transformation acting on the first index only, we get

$$
T_{n_{1}, \ldots, n_{d}} \rightarrow T_{n_{1}, \ldots, n_{d}}+\sum_{m} \epsilon_{n_{1} m} T_{m, \ldots, n_{d}}
$$

with $\epsilon=-\epsilon^{\dagger}$, leading to the functional equation called the Ward identity given by

$$
\begin{aligned}
& \sum_{\vec{n}_{\perp}, \vec{n}_{\perp}^{\prime}}\left\{\left(r_{k}(\vec{n})-r_{k}\left(\vec{n}^{\prime}\right)\right)\left[\frac{\partial^{2} W_{k}}{\partial J_{\vec{n}} \partial \bar{J}_{\vec{n}^{\prime}}}+\bar{M}_{\vec{n}} M_{\vec{n}^{\prime}}\right]\right. \\
& \left.\quad-\bar{J}_{\vec{n}} M_{\vec{n}^{\prime}}+\bar{M}_{\vec{n}} J_{\vec{n}^{\prime}}\right\}=0,
\end{aligned}
$$

where $\vec{n}_{\perp}=\left(n_{2}, \ldots, n_{d}\right)$. In the limit where the regulator goes to zero, the Ward identity is reduced to

$$
\sum_{\vec{n}_{\perp}, \vec{n}_{\perp}^{\prime}}\left(\bar{J}_{\vec{n}} M_{\vec{n}^{\prime}}-\bar{M}_{\vec{n}} J_{\vec{n}^{\prime}}\right)=0 .
$$

The meaning of this equation can be easily checked taking successive derivative with respect to the external sources. For instance, deriving with respect to $\partial^{2} / \partial J_{\vec{p}} \partial \bar{J}_{\vec{p}^{\prime}}$, we get

$$
\delta_{n_{1} p_{1}^{\prime}} \sum_{\vec{n}_{\perp}^{\prime}} \Gamma_{\vec{n}^{\prime} \vec{p}}^{(2)}=\delta_{p_{1} n_{1}^{\prime}} \sum_{\vec{n}_{\perp}} \Gamma_{\vec{p}^{\prime} \vec{n}}^{(2)}
$$

which is solved by $\sum_{\vec{n}_{\perp}^{\prime}} \Gamma_{\vec{n}^{\prime} \vec{p}}^{(2)} \propto \delta_{p_{1} n_{1}^{\prime}}$, where the coefficient is momentum independent. The same behavior remains true for all colors and we must have $\Gamma_{\vec{n} \vec{p}}^{(2)}=K \delta_{\vec{n} \vec{p}}$ where $K$ is an arbitrary constant. To be more precise let us notify the reader that the Ward identity arises from the $U(N)^{\times d}$ symmetry and ensures that the effective vertices inherit this invariance as well. The $r_{k}$-dependent term in (6) 
introduces a momentum dependence, providing a nonzero value for the momentum derivative of effective vertex functions. Moreover, this derivative may be expressed, at the leading order, in terms of a generalized trace invariant function, and meaning that such a truncation strongly violates the Ward identity. Obviously, let us consider once again the derivative with respect to $\partial^{2} / \partial J_{\vec{p}} \partial \bar{J}_{\vec{p}^{\prime}}$. We get, after some straightforward manipulations,

$$
\begin{aligned}
& \sum_{\vec{n}_{\perp}, \vec{n}_{\perp}^{\prime}}\left\{\left(r_{k}(\vec{n})-r_{k}\left(\vec{n}^{\prime}\right)\right)\left[G_{\vec{n} \vec{q}}^{(2)} \Gamma_{\vec{q} \vec{q}^{\prime} \vec{p} \vec{p}^{\prime}}^{(4)} G_{\vec{q}^{\prime} \vec{n}^{\prime}}^{(2)}\right]\right. \\
& \left.\quad+\delta_{\vec{n} \vec{p}} \Gamma_{k, \vec{p}^{\prime} \vec{n}^{\prime}}^{(2)}-\delta_{\vec{n}^{\prime} \vec{p}^{\prime}} \Gamma_{k, \vec{n} \vec{p}}^{(2)}\right\}=0 .
\end{aligned}
$$

Let us then simplify this expression. First, due to the momentum conservation along internal faces, we must have $\Gamma_{\vec{n} \vec{p}}^{(2)}=\gamma_{k}^{(2)}(\vec{p}) \delta_{\vec{n} \vec{p}}$. In the same way $G_{\vec{n} \vec{p}}^{(2)}=$ $g^{(2)}(\vec{p}) \delta_{\vec{n} \vec{p}}$. Moreover, at the leading order in the large $N$ limit, only the melonic diagram has to be retained for computing the effective loop in the right-hand side $[78,79,81]$. Note with this respect that disconnected pieces do not contribute, as pointed out in [81]. The melonic contribution $\Gamma_{\vec{q} \vec{q}^{\prime} \vec{p} \vec{p}^{\prime}}^{(4)}$ must have the following structure:

$$
\Gamma_{\vec{q} \vec{q}^{\prime} \vec{p} \vec{p}^{\prime}}^{(4)}=\sum_{i=1}^{d} \Gamma_{\vec{q} \vec{q}^{\prime} \vec{p} \vec{p}^{\prime}}^{(4),}
$$

with

$$
\Gamma_{\vec{q} \vec{q}^{\prime} \vec{p} \vec{p}^{\prime}}^{(4, i)}=2 \pi_{k}^{(2)}\left(p_{i}, q_{i}\right) \operatorname{Sym} W_{\vec{q} \vec{q}^{\prime} \vec{p} \vec{p}^{\prime}}^{(i)},
$$

and $\operatorname{Sym} W_{\vec{q} \vec{q}^{\prime} \vec{p} \vec{p}^{\prime}}^{(i)}:=W_{\vec{q} \vec{q}{ }^{\prime} \vec{p} \vec{p}^{\prime}}^{(i)}+W_{\vec{q} \vec{p}^{\prime} \vec{p} \vec{q}^{\prime}}^{(i)}$, where

$$
W_{\vec{q} \vec{q}^{\prime} \vec{p} \vec{p}^{\prime}}^{(i)}=\delta_{q_{i} p_{i}^{\prime}} \delta_{p_{i} q_{i}^{\prime}} \prod_{j \neq i} \delta_{q_{j} q_{j}^{\prime}} \delta_{p_{j} p_{j}^{\prime}}
$$

Taking $\vec{n}_{\perp}=\vec{n}_{\perp}^{\prime}$, we get, for $n_{1}=n_{1}^{\prime}+1$,

$$
r_{k}(\vec{n})-r_{k}\left(\vec{n}^{\prime}\right)=\left.\frac{1}{k} \frac{d}{d x_{1}} r_{k}(\vec{n})\right|_{n_{1}=k x_{1}}+\mathcal{O}(1 / k),
$$

where, for large $k$, we have introduced the continuous variable $x_{1}=n_{1} / k$. Note that $r_{k}(\vec{n})$ is assumed to be a function of $\vec{n} / k$. Therefore, keeping only the leading-order terms in the large $k$ limit, and setting $\vec{p}_{\perp}=\vec{p}_{\perp}^{\prime}=\overrightarrow{0}_{\perp}$, $p_{1}=n_{1}, p_{1}^{\prime}=n_{1}^{\prime}$ and finally $n_{1}=1, n_{1}^{\prime}=0$, we obtain

$$
2 \pi_{k}^{(2)}(0,0) \mathcal{L}_{2}=-\frac{d}{d x_{1}} \gamma_{k}^{(2)}(\overrightarrow{0}),
$$

with

$$
\mathcal{L}_{p}:=\sum_{\vec{n}_{\perp}} \frac{d r_{k}}{d x_{1}}\left(\vec{n}_{\perp}\right)\left(g^{(2)}\right)^{p}\left(\vec{n}_{\perp}\right)
$$

where we used the notation $f\left(\vec{n}_{\perp}\right) \equiv f\left(0, n_{2}, \ldots, n_{d}\right)$. The first derivative of the 2-point function, therefore, may be expressed only in terms of trace invariant functions, up to $1 / k$ correction. Interestingly we have the formal relation between this equation and the flow equation (1). As the flow equation dictates how the coupling changes when the scale changes, the Ward identity dictates how the coupling changes in momentum space. The existence of the momentum-dependent flow equation dictated by the Ward identity has the same origin as the scale-dependent flow equation which is dictated by the Wetterich equation such that the unitary symmetry breaking is provided by the regulator. In the next subsection, we will briefly recall what we call the local potential and dimension for RTM, and in the next section we will show that the Ward identities are strongly violated for such a local potential, except for a finely adjusted regulator, keeping the $r_{k}$-dependent term on the Ward identities as small as possible.

To conclude this section, note that we focus on regulators of the form

$$
r_{k}(\vec{n})=Z f\left(\frac{\sum_{i} n_{i}}{k}\right),
$$

where $Z$ is the wave function renormalization and $f(x)$ is assumed to be derivable and continuous, and satisfies the following criteria:

(1) $f(x) \rightarrow 0$ for $x \rightarrow \infty$,

(2) $f(x) \rightarrow \infty$ for $x \rightarrow 0$.

Note that in order to make simple analytic calculations, we focus on the Litim's regulator [91]:

$$
f(x)=\left(\frac{d}{x}-1\right) \theta\left(1-\frac{x}{d}\right),
$$

where $d$ is the rank of the tensor and $\theta$ the Heaviside step function. Particularly, in the following paper, we consider $d=3$.

\section{B. Locality, dimensionality, and melonic diagrams}

RTMs are nonlocal theories by construction. Tensorial invariants, whose connected components are called $b u b$ bles, are obtained as the product of the same number of $T$ and $\bar{T}$ fields, contracting their indices pairwise, the index $n_{i}$ of a field $T$ being contracted with the index $n_{i}$ of a field $\bar{T}$, ensuring unitary invariance by construction. Usually, these tensorial invariants are pictured as $d$-colored bipartite regular graphs (of rank $d$ ), where black and white nodes are respectively $T$ and $\bar{T}$ tensors, and the $d$ colored half edges hooked to them represent their $d$ indices. The different following paths which the edges are linked correspond to the invariant contractions. As an example, 

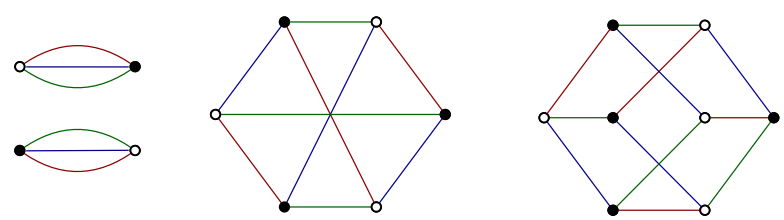

FIG. 1. Some examples of tensorial invariant in rank 3. The second and third (from left to right) are bubbles.

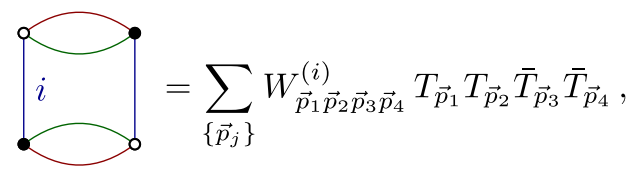

where $W^{(i)}$ has been defined in (11). Some examples for rank 3 are pictured in Fig. 1. The classical action $S(T, \bar{T})$ is assumed to be a local function, admitting an expansion as a sum of bubbles or product of bubbles, weighted by coupling constants $g_{b}$, labeled by the $d$-colored graph $b$.

The connected $2 N$-points functions may be expanded in powers of couplings, and Feynman amplitudes are indexed by Feynman graphs obtained from Wick's theorem. Such a typical diagram is provided in Fig. 2. Note that conventionally we associate the color 0 to the dotted edges. The only change is that Feynman graphs are enriched structures with respect to ordinary graphs, and correspond to the sets of vertices, edges, and faces. Before giving details of the calculation of the canonical dimension, let us recall the definition of face:

Definition 1. A face is a bicolored cycle, indexed by a couple $(i j)$. Such a cycle may be open (open face) or closed (closed face).

The scale behavior is required in the FRG and the scaling means a certain dependence on the number of size $N$ of the component-or equivalently on the dependence on $k$ in the RG flow viewpoint. For RTM, this terminology arises from the existence of a power counting. Indeed, the $1 / N$ expansion ensures that, up to a certain rescaling of the coupling constant,

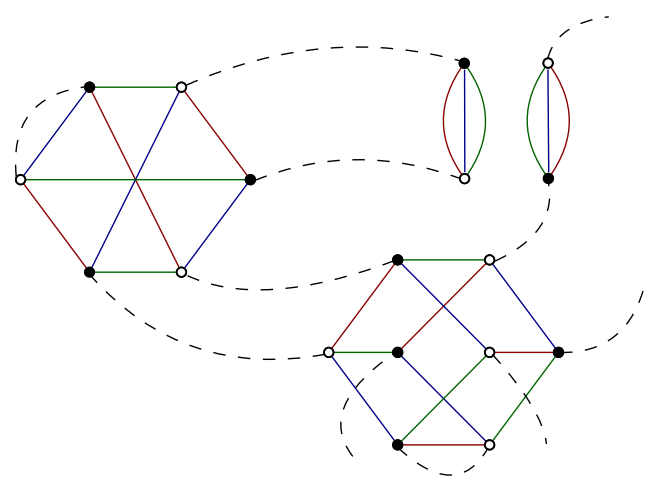

FIG. 2. A typical Feynman diagram for a three vertex amplitude with four external edges. The dotted edges correspond to Wick contractions.

$$
g_{b}=\bar{g}_{b} N^{\alpha(b)}
$$

then the Feynman amplitude $A(G)$ for the vacuum Feynman graph $G$ scales as $A(G) \sim N^{d-\frac{2}{(d-1) !} \varpi(G)}$, where $\varpi(G)$ is the Gurau degree given by the following definition:

Definition 2. Let $G$ be a $k$-colored bipartite regular graph with $|F|$ faces and $p$ black nodes. The Gurau degree $\varpi(G)$ is defined as

$\frac{2}{(k-2) !} \varpi(G)=\frac{(k-1)(k-2)}{2} p+(k-1)-|F|$.

The proper rescaling, for connected tensorial invariants, is given by [18-20]

$$
\alpha(b)=d-1-\frac{2}{(d-2) !} \varpi(b) .
$$

The leading order diagrams, for which $\varpi(G)=0$, are said to be melonic. For a melonic vacuum diagram, all the interaction bubbles have to be melonic as well. Melonic diagrams may be defined recursively, and their continuum limit corresponds to a branched polymer phase [21]. Melonic diagrams with external edges are defined in the same way. They correspond to the leading order diagrams in the $1 / N$ expansion, and follows a similar recursive definition. They can be obtained from vacuum diagrams by deleting some dotted edges. Locality, in a RTM, as in tensor field theories, is defined from tensor invariance.

Definition 3. The bubbles, or sums of bubbles, are said to be ultralocals. Moreover, a sum of bubbles and product of bubbles is said to be local.

From this definition, an effective action builds as the sum of bubbles is said to be an ultralocal potential. By extension, a local potential involves only bubbles or products of them, including therefore disconnected pieces. The canonical dimension of the interaction arises from the scaling (20). It is convenient for RG applications to fix to 1 the scaling of the kinetic term $[74,75,80]$. This can be achieved by a rescaling of the fields $T \rightarrow N^{-\frac{d-1}{2}} T$, modifying the scaling (20) as

$$
\alpha^{\prime}(b)=d-1-\frac{d-1}{2} n(b)-\frac{2}{(d-2) !} \varpi(b) .
$$

We call canonical dimension this quantity, where $n(b)$ denotes the number of fields involved in the connected bubble $b$. The scaling for bubbles must be completed by the scaling law for disconnected invariants. Let us consider $h=b_{1} * b_{2}$, a disconnected tensorial invariant made with two bubbles $b_{1}$ and $b_{2}$, and we define the difference:

$$
\delta \alpha^{\prime}\left(b_{1} * b_{2}\right)=\alpha^{\prime}\left(b_{1} * b_{2}\right)-\alpha^{\prime}\left(b_{1}\right)-\alpha^{\prime}\left(b_{2}\right) .
$$

We fix $\delta \alpha^{\prime}(h)$ in accordance with the scaling dimension of the kinetic operator, which is set to be zero. To this end, 
we expect that the scaling of the different operators have to be such that, for any bubble $b$, there exists an optimal way to build a 2-point diagram max $G(b)$ whose amplitude $A(\max G)$ scales as $N^{0}$. The same requirement must be true for interactions made with disconnected pieces. Noting that, with respect to a connected graph, we lose a bicolored cycle merging the two connected components $b_{1}$ and $b_{2}$. The resulting graph $G(h)$ can be connected or not. For $G(h)$ being disconnected, we can factorize $\max G(h)=$ $\max G\left(b_{1}\right) \max \bar{G}\left(b_{2}\right)$, where we assumed that it corresponds to the optimal contraction, and denoted as $\bar{G}\left(b_{2}\right)$ the vacuum graph obtained from $b_{2}$. From the definition of the amplitude of the graph, we must have

$$
\begin{aligned}
A(\max G(h)) & \sim A\left(\max G\left(b_{1}\right)\right) \times A\left(\bar{G}\left(b_{2}\right)\right) \\
& =\mathcal{O}(1) \times A\left(\bar{G}\left(b_{2}\right)\right) \sim N^{d},
\end{aligned}
$$

where we used the scaling theorem for vacuum diagrams. In the case where $\bar{G}(h)$ is connected, the above factorization is not held. However, it is not difficult to check that such a contribution has to be less relevant, some internal faces being discarded to ensure connectivity. The optimal counting is therefore $A(\max G(h)) \sim N^{d}$, enforcing one to choose (optimally) $\delta \alpha^{\prime}(h)=-d(k-1)$. In the same way, for a disconnected interaction to build as $k$ bubbles, $h_{k}=b_{1} * b_{2} * \cdots * b_{k}$, we require $\delta \alpha^{\prime}\left(h_{k}\right)=-d(k-1)$, and finally

$$
\alpha^{\prime}\left(b_{1} * \cdots * b_{k}\right)=\sum_{\ell=1}^{k} \alpha^{\prime}\left(b_{\ell}\right)-d(k-1),
$$

which can be conveniently rewritten as

$$
\begin{aligned}
\alpha^{\prime}\left(b_{1} * \cdots * b_{k}\right)= & (d-1)-\frac{d-1}{2} \sum_{i} n\left(b_{i}\right) \\
& -\frac{2}{(d-2) !} \sum_{i} \varpi\left(b_{i}\right)-(k-1) .
\end{aligned}
$$

In the rest of this paper, we denote by $d_{g}$ the scaling dimension for the coupling $g$. To conclude this part, let us mention that this scaling holds only at zero order around the Gaussian fixed point, as in ordinary quantum field theory; and the first quantum deviations from this Gaussian counting arise from the anomalous dimension. In the present case, it is played by the kinetic prefactor, which we denote by $Z(k)$ (do not confuse this with the partition function $Z_{k}$ ), and plays the role as an effective mass. It is suitable to set the normalization such that this coefficient remains equal to 1 along the flow (this is, moreover, a condition to get fixed points). We thus rescale $T$ as $Z^{-1 / 2}(k) T$, and we finally define the renormalized couplings as

$$
\bar{g}_{b}=g_{b} Z^{-n(b) / 2}(k) N^{-\alpha^{\prime}(b)} .
$$

\section{Product of distributions and regularization}

In this manuscript, we will consider the sharp regulators of the form

$$
f(x)=g(x) \theta(1-x),
$$

on which we intend to give the meaning of the integrals of the form

$$
I_{n, p}=\lim _{\Lambda \rightarrow \infty} \int_{0}^{\Lambda} \frac{x^{n} f^{\prime}(x)}{(1+f(x))^{p}} .
$$

Note that this integral appears throughout this paper in the computation of the Wetterich flow equation as well as in the explicit relation of the Ward identities. The regulator (26) introduces a sudden cut in the space of the indices and this is suitable for field theories without background. The Litim regulator (16), commonly used in the functional renormalization group (FRG) literature is an example, with $g(x)=1 / x-1$. However, for more general choices of $g(x)$, the exact flow equation (1) cannot be used without a prescription for the product $\delta(x) \theta(x)$; and the integral (27) does not make sense.

There are essentially two ways to solve this ambiguity, and we refer respectively to them as scheme 1 " $\left(S_{1}\right)$ and scheme 2" $\left(S_{2}\right)$ :

In the first scheme, $S_{1}$, which is the most used in the literature [98-100], we solve formally the ambiguity arising in the ill-defined integral (26) by considering the Heaviside distribution as the limit of regular functions $\theta(x)=\lim _{a \rightarrow 0} \theta_{a}(x)$, for which the integral makes sense. A basic example is

$$
\theta_{a}(x)=\frac{1}{a \sqrt{\pi}} \int_{-\infty}^{x} e^{-y^{2} / a^{2}} d y .
$$

This can also be achieved by a series of functions which converge weakly towards the Heaviside distribution:

$$
\Theta_{n}(x):=\frac{x^{n}}{e^{x^{n}}-1}, \quad \lim _{n \rightarrow \infty} \Theta_{n}(x)=\theta(x) .
$$

This allows one to, formally, remove the ambiguity which appears by rewriting the products like $\delta(x) \theta(x)$. This can be achieved formally from a simple partial integration of (26). Assuming that $f(x)$ is an ordinary regular function rather than a distribution, we have trivially

$$
I_{n, p}=\lim _{\Lambda \rightarrow \infty}\left[\frac{n}{p-1} \int_{0}^{\Lambda} \frac{x^{n-1} d x}{(1+f(x))^{p}}-\frac{1}{p-1} \Lambda^{n}\right],
$$

where we assumed that $f(x)$ vanishes for large $x$, which is satisfied for a regulator. The two expressions (27) and (30) are equivalent when $f$ is considered as a function. However, only the last one is well defined when $f$ is a distribution as (26). Then, we can use this form as a definition of the 
ill-defined product $f^{\prime}(x) f(x)$ for the computation of the integral. We get explicitly

$$
I_{n, p}=\frac{n}{p-1} \int_{0}^{\alpha} \frac{x^{n-1} d x}{(1+g(x))^{p}}-\frac{1}{p-1} \alpha^{n} .
$$

In the second scheme, $S_{2}$, we remember that the derivative $\dot{r}_{k}$ is a formal operation. Indeed, $k$ must be an integer and $\dot{r}_{k}$ becomes a formal derivative only in the large $k$ limit. For finite $k$, it must be a finite difference:

$$
\dot{r}_{k}(x) \equiv r_{k+1}(x)-r_{k}(x),
$$

and there is no ambiguity with the sums like

$$
\mathcal{S}_{n, p}=\sum_{\vec{n}=\overrightarrow{0}}^{\infty}\left(\sum_{i} n_{i}\right)^{n} \frac{f\left(\frac{\sum_{i} n_{i}}{k+1}\right)-f\left(\frac{\sum_{i} n_{i}}{k}\right)}{\left(1+f\left(\frac{\sum_{i} n_{i}}{k}\right)\right)^{p}} .
$$

Introducing the parameter $\epsilon:=1 / k$, the ambiguity in the formal expression of the product $f^{\prime}(x) f(x)$ in the integral (27) then reads as

$$
\int x^{n} \frac{\theta(1+\epsilon-x)-\theta(1-x)}{(1+g(x) \theta(1-x))^{p}} d x .
$$

In the interval $x \in[1,1+\epsilon]$, we must have $\theta(1-x)=0$; and in the continuum limit $\epsilon=0$, we may set

$$
\int x^{n} \frac{\theta(1+\epsilon-x)-\theta(1-x)}{(1+g(x) \theta(1-x))^{p}} d x \rightarrow \epsilon \int x^{n} \frac{\delta(1-x)}{\left(1+0^{\prime \prime}\right)^{p}} d x .
$$

Note, however, that we can make another choice for the finite difference (32). The following example holds:

$$
\dot{r}_{k}(x) \equiv r_{k}(x)-r_{k-1}(x),
$$

so that the integral (37) becomes

$$
\int x^{n} \frac{\theta(1-x)-\theta(1-\epsilon-x)}{(1+g(x) \theta(1-x))^{p}} d x .
$$

For the ordinary regular functions, there is no difference between left and right derivatives; however, in this case, the two definitions are not equivalent at all. In the interval $x \in[1-\epsilon, 1]$, we must have $\theta(1-x)=1$, so that with this definition the integral becomes

$$
\int x^{n} \frac{\theta(1-x)-\theta(1-\epsilon-x)}{(1+g(x) \theta(1-x))^{p}} d x \rightarrow \epsilon \int x^{n} \frac{\delta(1-x)}{(1+g(x))^{p}} d x .
$$

The convention (38) has been used in the case of matrix models in [80]. The convention (38) holds for the matrices theories, but becomes pathological for tensors models, with respect to the operations that we will consider for our regulator. ${ }^{1}$ Therefore, we keep the second convention given by Eq. (38) i.e., the scheme 2.

Note that except for the case where $g(1)=0$, which corresponds to the Litim regulator, the two definitions are nonequivalents. Moreover let us notify the reader that there is another way to consider the scheme $S_{1}$ i.e., we can make the restriction on $S_{1}$ which we will denote by $S_{1}^{\prime}$, by starting directly with a regularized expression for the regulator. Indeed, we can use a regularized expression for the Heaviside distribution, $\theta_{a}(x)$, such that $\lim _{a \rightarrow 0} \theta_{a}(x)=\theta(x)$, to compute the integral (27) [98]. Indeed, to solve the ambiguity, we have to provide a sense to the $\operatorname{limit}_{\lim _{a \rightarrow 0}} \theta_{a}^{\prime}(1-x) G\left(\theta_{a}(1-x)\right)$, for some regular function $G$. This can be achieved, for instance, using the identity

$$
\theta_{a}^{\prime}(1-x) G\left(\theta_{a}(1-x)\right)=\frac{d}{d x} \int_{\theta_{a}(1-x)}^{A} G(y) d y,
$$

where we consider the upper bound $A$, such that the integral exists in the limit $a \rightarrow 0$. Taking the limit, it is not difficult to show that

$$
\theta_{a}^{\prime}(1-x) G\left(\theta_{a}(1-x)\right) \rightarrow \delta(1-x) \int_{0}^{1} G(y) d y .
$$

From some elementary algebraic manipulations, it is easy to check that this regularization scheme provides exactly the same expression as (35); therefore $S_{1} \sim S_{1}^{\prime}$.

In the next sections, we will use these two regularization schemes, and show explicitly that the corresponding results are strongly dependent on them. This is, once again, an artifact of the symmetry breaking required to construct the RG flow.

\section{PROGRESSIVE LOCAL TRUNCATIONS AND MODIFIED REGULATOR}

In this section, we construct the approximate solutions of RG equation (1) using local potential approximation. We start with melonic approximation, keeping only connected diagrams. We show that the Ward identity violation can be improved at first order, from an appropriate modification of the Litim regulator without losing its optimal character in the sense of Litim [91,92]. We then discuss the essential role played by the disconnected diagrams and show that a melonic ultralocal truncation of arbitrary order cannot reach the exact value of the critical exponent for the single relevant direction of the non-Gaussian fixed point. Note that we keep the notation $d$ for the rank of the tensor, without specifying the value of $d$, to highlight the origin of

\footnotetext{
${ }^{1}$ See the next section. Using the first convention, we do not find any solution which makes $\alpha$ such that $\mathcal{L}_{2}$ vanishes.
} 
the contribution, but ultimately we only focus on $d=3$. Moreover, we focus on the symmetric phase, and we expand beta functions around vanish means fields $M$ and $\bar{M}[78-86]$.

\section{A. Quartic truncation}

Let us start with a quartic local truncation:

$$
\Gamma[M, \bar{M}]=Z(k) \Longleftrightarrow+g(k) \sum_{i=1}^{d} \sum_{0}
$$

The flow equations can be obtained from the exact flow equation (1) taking successive derivatives with respect to $M$ and $\bar{M}$ fields. The flow equation for $\eta(k)=\partial_{k} \ln (Z(k))$ can be deduced taking the derivative with respect to $\partial^{2} / \partial M_{\vec{p}} \partial \bar{M}_{\vec{q}}$, and setting $\vec{p}=\vec{q}=\overrightarrow{0}$. Graphically, at leading order in $k$ for large $k$, we get an equation of the form

$$
\dot{Z}=-2 g(k) \sum_{i=1}^{d}
$$

where the dotted edge corresponds to contraction with respect to $\dot{r}_{k}\left(G^{(2)}\right)^{2}$. Moreover, the dot is defined as $\dot{X}=k \partial X / \partial k$-the factor 2 counting the number of derivatives relevant at the leading order in $k$. The flow equation for $g(k)$ may be easily deduced in the same way:

$$
d \times \dot{g}=4 g^{2}(k) \sum_{i=1}^{d} \bigcap_{0}^{i} \bigcap^{i},
$$

where once again the dotted edges represent contractions with propagators $G^{(2)}$ and $\dot{r}_{k}\left(G^{(2)}\right)^{2}$. In principle, we identify the terms on both sides of the flow equations weighting the same boundary graphs. Let us recall the definition of a boundary graph:

Definition 4. Let $G$ be a $d+1$-colored Feynman graph (including edges of color 0 ) and $\mathcal{F}_{0}$ the set of external faces of type $0 i$ for $i \in(1, \ldots, d)$.

Let $f_{0 i} \in \mathcal{F}_{0}$. The boundary graph of $f_{0 i}$ denoted by $\partial f_{0 i}$ is the set of bicolored edges of type 0 and $i$ building the cycle $f_{0 i} . i$ is called the color of the boundary / partial $f_{0 i}$.

The boundary graph of $G, \partial G$ is a $d$-colored graph (connected or not) built as the set of nodes hooked to external edges and of the boundaries of external faces, such that each boundary $\partial f_{0 i}$ is identified to a single edge of color $i$.

Figure 3 provides an illustration of a such boundary graph. Equations (42) and (43) can be easily solved using

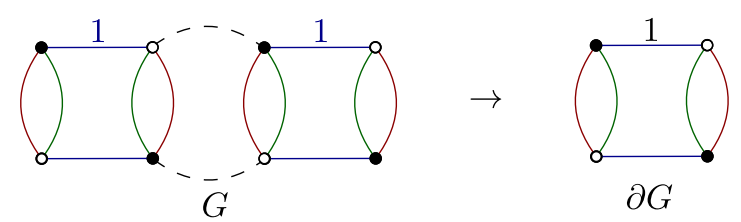

FIG. 3. On the left a Feynman graph $G$, and the corresponding boundary graph $\partial G$ on the right.

the Litim's regulator. However, the Ward identity (13) is strongly violated using the Litim's regulator (16) with the truncation (41). Indeed, the left-hand side of Eq. (13) reads as

$$
-\frac{2}{d} g(k) \sum_{\vec{n}_{\perp}} \Theta\left(k-\frac{\sum_{i=2}^{d} n_{i}}{d}\right) \sim-\frac{2}{d} g(k)(k \cdot d)^{d-1},
$$

where we used the renormalization condition $\pi_{k}^{(2)}=g(k)$. This term is therefore of order $\bar{g}(k)$, which is in accordance with the expected result. The problem is heuristically pictured in Fig. 4 where the plane $\mathcal{M}$ represents the largest theory space, including nonlocal (momentum-dependent) couplings. The RG flow thus may be viewed as a map $\mathrm{R}: \mathbb{R} \rightarrow \mathcal{M}$, corresponding to the trajectory relaying different points of the theory space at different times $t_{1}=\ln \left(k_{1}\right)$, $t_{2}=\ln \left(k_{2}\right) \cdots$. Starting with a purely local truncation, involving only bubbles or products of bubbles, the flow does not remain along the local trajectory (the red dotted arrow) but is derived toward a nonlocal region. This is a consequence of the Ward identity (13). The derivative $d \gamma / d x_{1}$ is nonzero even if the original truncation involves only the local terms. To solve this difficulty, and following [80], we try to modify the windows of allowed momenta, such that

$$
f(x)=\left(\frac{d}{x}-1\right) \theta\left(\alpha-\frac{x}{d}\right)
$$

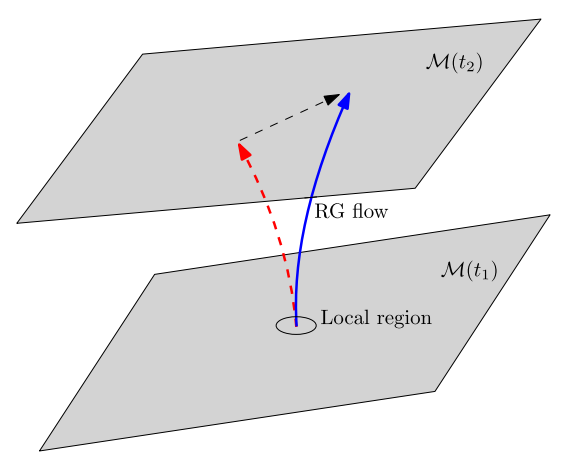

FIG. 4. Heuristic picture of the RG flow. Starting from a purely local region (corresponding to local truncation), the RG flow (the solid blue arrow) derives toward a nonlocal region instead of remaining along the red trajectory corresponding to the local flow, due to the Ward identities. 
and then fine-tune $\alpha$ in such a way that the boundary conditions on $f$ remain held, and that (44) vanishes. Indeed, for $\alpha \neq 1$, the formal derivative of the Heaviside function provides a nonvanishing contribution proportional to $\delta\left(\alpha-\frac{x_{\perp}}{d}\right)$ :

$$
\left.\frac{\partial f}{\partial x_{1}}\right|_{x_{1}=0}=-\frac{d}{x_{\perp}^{2}} \theta\left(\alpha-\frac{x_{\perp}}{d}\right)-\frac{1}{d}\left(\frac{1}{\alpha}-1\right) \delta\left(\alpha-\frac{x_{\perp}}{d}\right),
$$

leading to an undefined product as $\delta(\alpha-x) \theta^{n}(\alpha-x)$. The regularization schemes proposed in the previous section aim to solve this ambiguity. Using the continuum limit, for $k \gg 1$ to replace sums by integrals, we have to compute integrals of the form

$$
J=\int d x_{1} d x_{2} \theta\left(\alpha-x_{1}-x_{2}\right) f\left(x_{1}+x_{2}\right),
$$

which can be easily computed by elementary algebraic manipulations, see the Appendix. We get

$$
J=\int_{0}^{\alpha} d x x f(x) .
$$

Using integral approximation (valid for $d=3$ ),

$$
\sum_{\vec{n}_{\perp}}\left(\frac{\sum_{i=2}^{d} n_{i}}{k}\right)^{p} \theta\left(k-\frac{\sum_{i=2}^{d} n_{i}}{d}\right) \approx k^{2} \frac{d^{p+2}}{p+2},
$$

and the regularization scheme $S_{1}$, the condition $\mathcal{L}_{p}=0$ reads as

$$
\alpha-\frac{1}{p} \alpha^{p}=0,
$$

and setting $p=2$, we get $\alpha=2$. Using the scheme $S_{2}$, and the well-known identity $\partial \theta(\alpha-x) / \partial \alpha=\delta(\alpha-x)$, we get straightforwardly a unique solution $\quad \alpha=3 / 2^{2} \quad \alpha=3 / 2$ coming from the equation

$$
-\frac{1}{2} \alpha^{2}-\alpha^{2}(1-\alpha)=0 .
$$

In the hope of deriving another solution for this problem, we can attempt to try the modification $f(x) \rightarrow f(x)+$ $\alpha x f(x)$. However, all such solutions are in conflict with the positivity requirement of the effective propagator in the interval $x \leq d$, which introduces some singularities and, therefore, we discarded them. Finally, let us add an important remark about this derivation. The reader may have some doubts about the use of the truncation (41) to compute the integral on the left-hand side of the Ward identity. However, we have to keep in mind (and it is clear

\footnotetext{
${ }^{2}$ In rank $d$, it may be easily checked that $\alpha=d /(d-1)$.
}

for the regulator that we have chosen) that the windows of momenta relevant for the computation of this integral, provided by the distribution $\partial f / \partial x_{1}$, are exactly the same $\left(x_{\perp} \leq \alpha d\right)$ as the one provided by $\dot{r}_{k}$ into the flow equation [see Eq. (51)]. Therefore, using the truncation to compute the sum on the right-hand side of the Ward identity is not an additional approximation. It is the same as using the truncation to solve the flow equation.

Equations (42) and (43) can be explicitly computed using the regulator (45):

$$
\begin{aligned}
\dot{r}_{k}(k x)= & Z\left[\eta\left(\frac{d}{x}-1\right)+\frac{d}{x}\right] \theta\left(\alpha-\frac{x}{d}\right) \\
& +Z(1-\alpha) \delta\left(\alpha-\frac{x}{d}\right),
\end{aligned}
$$

where we used $g(x) \delta(\alpha-x)=g(\alpha) \delta(\alpha-x)$. Using the sum (48), we get

$$
\eta=-6 g(k) \frac{k^{d-1}}{Z^{2}}\left[\eta\left(l_{-1,2}-l_{0,2}\right)+t_{-1,2}+(1-\alpha) \partial t_{0,2}^{(S)}\right],
$$

and

$$
\dot{g}=4 g^{2}(k) \frac{k^{d-1}}{Z^{2}}\left[\eta\left(\iota_{-1,3}-\iota_{0,3}\right)+l_{-1,3}+(1-\alpha) \partial \iota_{0,3}^{(S)}\right],
$$

where, in the large $k$ limit,

$\iota_{p, q}:=\int_{0}^{\infty} d^{d-1} x|x|^{q+p} \frac{\theta(d \alpha-x)}{d^{q+p}}=\frac{(\alpha)^{p+q+2} d^{2}}{p+q+2}$.

The explicit expression for $\partial \iota_{p, q}^{(S)}$, however, depends on the regularization scheme $S=S_{1}$ or $S=S_{2}$. For $S=S_{1}$ we have

$$
\partial \iota_{p, q}^{\left(S_{2}\right)}=d^{2} \frac{\alpha^{2}}{p-1} \frac{1}{1-\alpha}
$$

and for $S=S_{2}$,

$\partial l_{p, q}^{\left(S_{2}\right)}=\int_{0}^{\infty} d^{d-1} x|x|^{q+p} \frac{\delta(d \alpha-x)}{d^{q+p}}=(\alpha)^{p+q+1} d^{2}$,

where the norm $|$.$| is defined as |x|:=\sum_{i} x_{i}$. In terms of the renormalized couplings (25), defining $\beta_{g}:=\dot{\bar{g}}$ the previous equations read in the scheme $S_{1}$ as

$\beta_{g}^{\left(S_{1}\right)}=2(1-\eta) \bar{g}+12 \alpha^{4} \bar{g}^{2}\left[3 \eta \frac{5-4 \alpha}{20}-\frac{3}{4}+\frac{3}{2 \alpha^{2}}\right]$,

where

$$
\eta^{\left(S_{1}\right)}:=\frac{36(3-2 \alpha) \alpha^{2} \bar{g}}{9 \alpha^{3}(3 \alpha-4) \bar{g}-2} .
$$


Using the scheme $S_{2}$,

$\beta_{g}^{\left(S_{2}\right)}=2(1-\eta) \bar{g}+12 \alpha^{4} \bar{g}^{2}\left[3 \eta \frac{5-4 \alpha}{20}+\frac{3}{4}+3(1-\alpha)\right]$,

where

$$
\eta^{\left(S_{2}\right)}:=-\frac{8}{9 \alpha^{3}(3 \alpha-4) \bar{g}-2}-4
$$

Once again, note that the two schemes are equivalents for $\alpha=1$. Equation $\beta_{g}=0$ can be exactly solved for arbitrary $\alpha$. In particular, note the following:

First, for $\alpha=1$ (standard Litim regulator), we get two fixed points, $g_{1} \approx-6.29$ and $g_{2} \approx-0.037$, with respective anomalous dimension and critical exponents ${ }^{3}$ :

$\eta_{1} \approx-4.14, \quad \theta_{1} \approx 12.3, \quad \eta_{2} \approx 0.81, \quad \theta_{2} \approx 2.39$.

The first fixed point has a very large critical exponent, and the anomalous dimension violates the regulator bound. ${ }^{4}$ Therefore, at this stage, we do not have confidence with this fixed point, which can be viewed as an artifact of the approximation.

Second, in the scheme $S_{2}$, for $\alpha=1.5$ we get two fixed points $g_{1}^{*} \approx 1.74$ and $g_{2}^{*} \approx 0.017$ with anomalous dimensions and critical exponents respectively:

$\eta_{1}^{*} \approx-4.33, \quad \theta_{1}^{*} \approx 17.0, \quad \eta_{2}^{*} \approx 0.57, \quad \theta_{2}^{*} \approx 2.26$.

The two fixed points have essentially the same characteristics as the fixed point $g_{1}$ and $g_{2}$ obtained using the Litim regulator, enforcing the confidence in the local truncation for the existence of this fixed point. The properties of the fixed point $g_{2}$ coincide with the ones of the relevant fixed point discovered in [101] using the same purely local truncation with the Litim's regulator. We see that the modified regulator with $\alpha=3 / 2$ slightly improves the result, the exact result being $\theta=d-2$ for $d$ is the rank of the tensor [101].

Finally, in the scheme $S_{1}$, for $\alpha=2$, we get again two fixed points, for $g_{1}^{* *} \approx-0.35$ and $g_{2}^{* *} \approx 0.005$, with characteristics

\footnotetext{
${ }^{3}$ We recall that critical exponents are defined as the opposite values of the stability matrix $A_{i j}:=\partial_{g_{i}} \beta_{j}$ evaluated at a given fixed point.

${ }^{4}$ The regulator has to be very large in the large $k$ limit. For the Litim regulator, taking into account the definition of $\eta$, we must have $r_{k} \sim k^{1+\eta}$ in the large $k$ limit, ensuring $\eta>-1$.
}

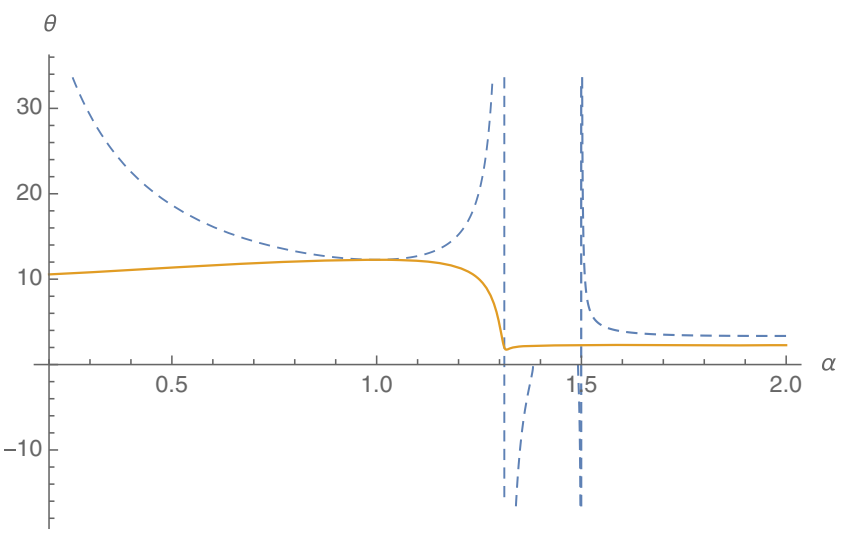

FIG. 5. Plot of $\theta(\alpha)$ for $\alpha \in[0.2,2]$, using scheme $S_{1}$ (blue dashed curve) and scheme $S_{2}$ (yellow solid curve).

$\eta_{1}^{* *} \approx-0.96 ; \quad \theta_{1}^{* *} \approx 4.96 ; \quad \eta_{2}^{* *} \approx 0.65 ; \quad \theta_{2}^{* *} \approx 3.35$.

The second fixed point $g_{2}^{* *}$ is reminiscent of the two fixed points $g_{2}$ and $g_{2}^{*}$, especially concerning the value of their anomalous dimensions, and may be interpreted as the fixed point governing the continuum limit corresponding to the double scaling. However, the first fixed point has the interesting property i.e., the anomalous dimension remains below the lower bound $\eta=-1$. Therefore, there is no reason before discarding it. The only reason may be that it seems to be very dependent on the scheme used to do the computation, but at this stage, there is no strong indication to privilege scheme $S_{1}$ regarding the scheme $S_{2}$. Usually, only the stability regarding higher truncations may provide a solid argument to keep or discard such a fixed point.

Nevertheless, stability for small variations of the regulator and the presence of singularities may provide a first indication about the quality of the regularization scheme. Figure 5 shows the dependence of the critical exponents for the second fixed point with $\alpha$, respectively for schemes $S_{1}$ and $S_{2}$. The blue curve (scheme $S_{1}$ ) is stable in the region $\alpha=1$, a possible indication of why the Litim regulator works well. ${ }^{5}$ It becomes stable also in the vicinity of $\alpha=2$, is a larger domain than for $\alpha=1$, and has better stability which is encouraging physically, despite the strong disagreement with the exact result $(\theta \approx 3.35$ when the exact value is $\left.\theta_{\text {extact }}=1\right)$ - a conclusion which has to be confirmed for higher truncations. However, between these two regions, the curve of $\theta$ has two singularities. In contrast, the yellow curve in the scheme $S_{2}$ does not has any singularity. It is stable on a long-range of values around $\alpha=1$; and after a continuous transition, becomes stable once again in the region $\alpha \approx 3 / 2$. Based on these elementary investigations, scheme $S_{2}$ seems to behave better than scheme $S_{1}$ (in

\footnotetext{
${ }^{5}$ Note, however, that the critical exponent is $\theta \approx 12$ for $\alpha=1$, a characteristic reminiscent of the fixed point $g_{1}$.
} 


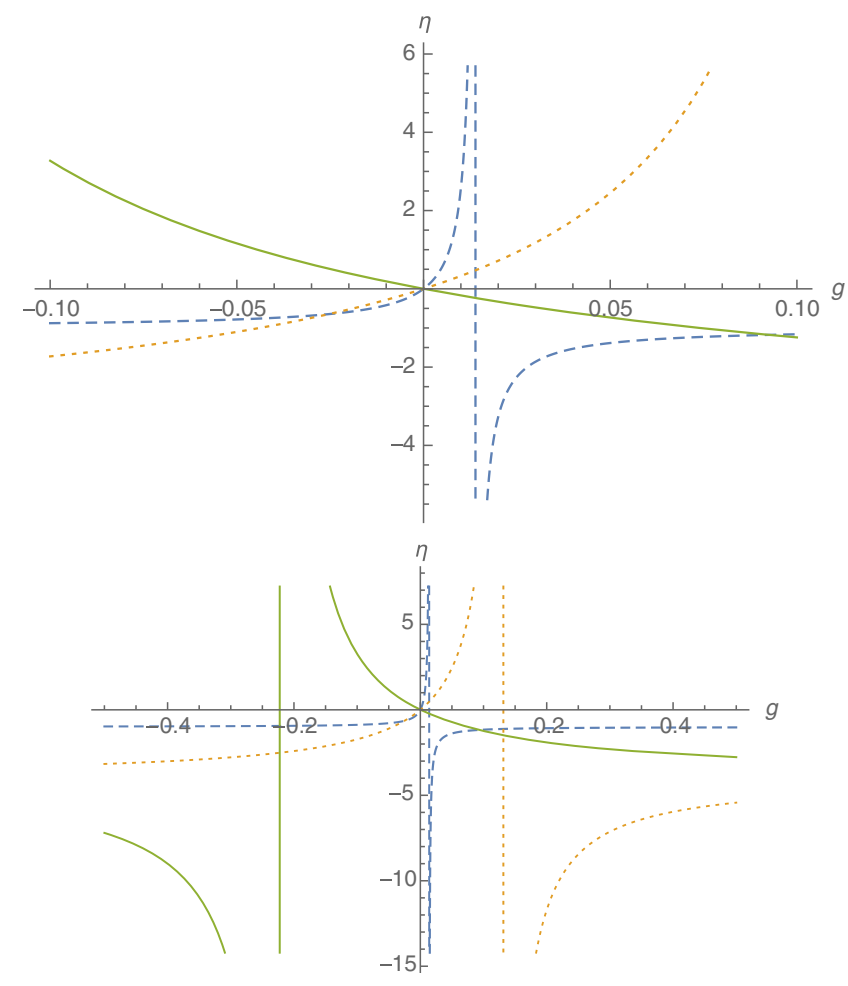

FIG. 6. Plot of the anomalous dimension in a short range of couplings around the Gaussian fixed point. For $\alpha=1$ (solid green curve), for $\alpha=2$ using scheme $S_{1}$ (blue dashed curve), and for $\alpha=1.5$ using scheme $S_{2}$ (dotted yellow curve).

the relevant range of values of $\alpha$ that we investigated); it is encouraging to take results arising from $S_{2}$ as reference. We will complete these conclusions in the next sections.

Figures 6 and 7 show respectively the anomalous dimensions and the $\beta$ functions for $\alpha=1, \alpha=2$, and $\alpha=3 / 2$, respectively using schemes $S_{1}$ and $S_{2}$. All the solutions are in quantitative accordance in the vicinity of the Gaussian fixed point, but differ quantitatively and qualitatively in a relatively large range of couplings, before finding a qualitative agreement for couplings of very large magnitude (see the second curve of Fig. 6). Note that all of the regularization schemes have a singularity in the vicinity of their zeros, in the negative region for $\alpha=1$ and in the positive region for $\alpha=2\left(S_{1}\right)$ and $\alpha=3 / 2\left(S_{2}\right)$. Note that the quality of the regularization scheme could be very dependent on the region that we consider. Indeed, we have seen that, for small couplings, regularization $S_{2}$ has a better behavior than $S_{1}$, which is also clear from the curves for $\eta$ and $\beta$, and have a singularity very closer to the Gaussian fixed point. However, the curve for $\eta$ shows that for the Litim regulator and $S_{2}$ approach with $\alpha=3 / 2$, the anomalous dimension becomes smaller than the lower bound $\eta=-1$ for couplings with large magnitude. In contrast, the value for the anomalous dimension using $S_{1}$ remains not so far from the bound in the positive region, and just above that in the negative region.

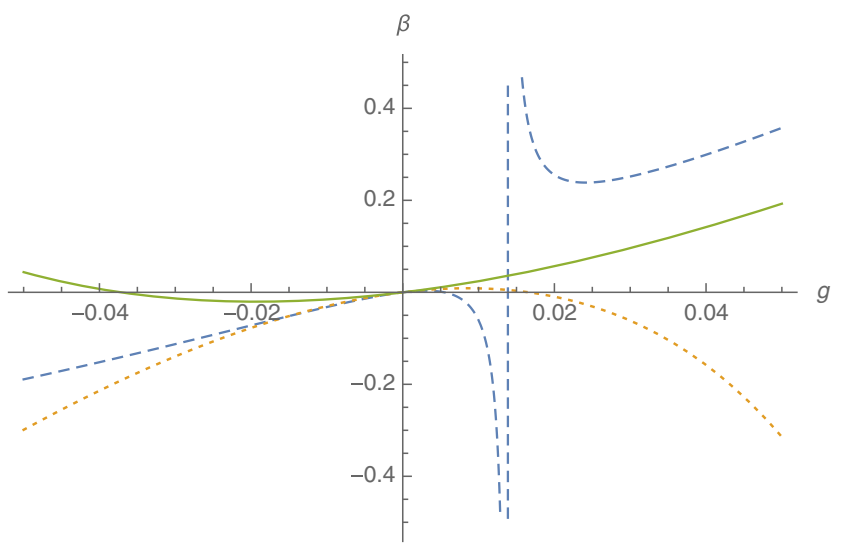

FIG. 7. Plot of the $\beta$ function for $\alpha=1$ (solid green curve), $\alpha=2$ using scheme $S_{1}$ (blue dashed curve), and $\alpha=1.5$ using scheme $S_{2}$ (dotted yellow curve).

It is not easy to say more only from quartic truncations, especially with the improvement coming from taking into account Ward identities in the construction of the local flow. This solution, however, takes into account only the first-order effects, the first derivative for the first Ward identity, involving only 4- and 2-point functions. A deeper investigation obviously should take into account higherorder effects. However, we will see in the next section that taking into account first-order effects already shows a clear improvement, mainly visible in the rapidity of the convergence of the results in high truncations.

\section{B. Octic truncations}

In this section we investigate higher order melonic truncations, taking into account sextic and octic couplings. Taking into account all the melonic connected couplings up to valence eight, we get, in the same notations as in the previous section,

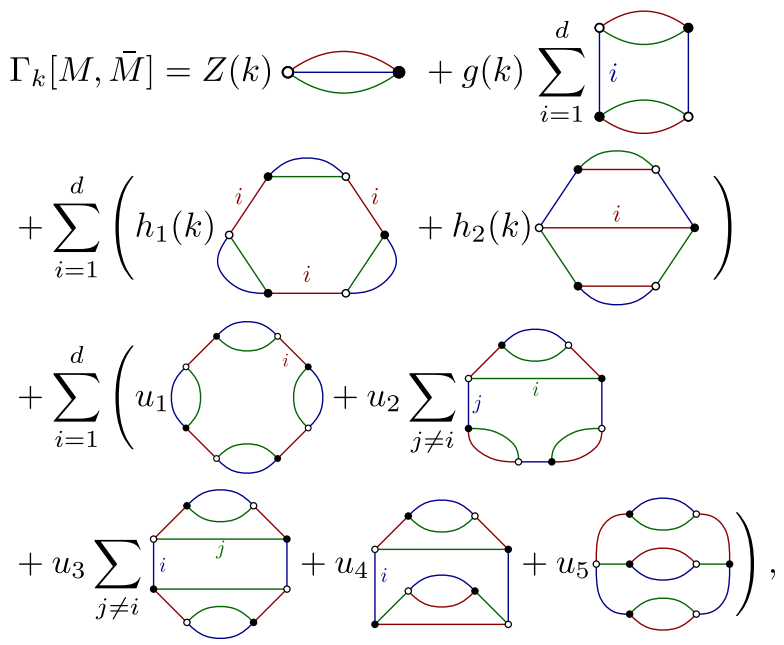

The flow equations for the couplings can be easily derived taking successive derivatives of the exact RG equation (1). 
Equation (42) remains unchanged. The equation for $\dot{g}$, however, receives sextic contributions, and becomes graphically

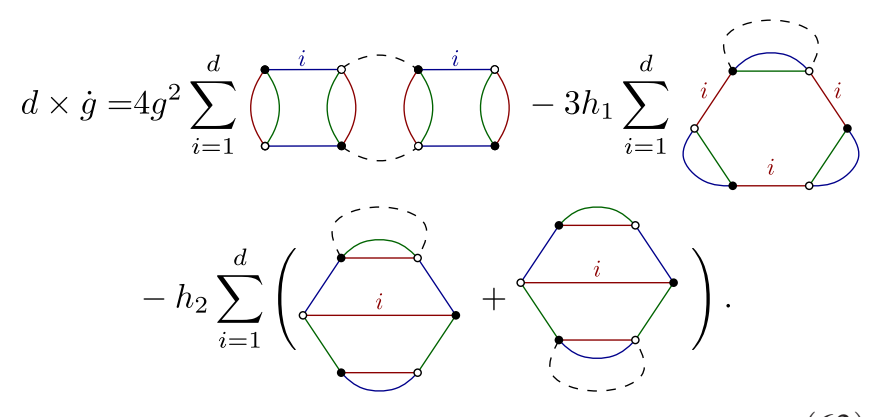

The flow equations of $h_{2}$ and $h_{1}$ can be derived in the same way, and we get

$$
\text { × } \dot{h}_{2}=4 g h_{2} \sum_{i=1}^{d}
$$

and

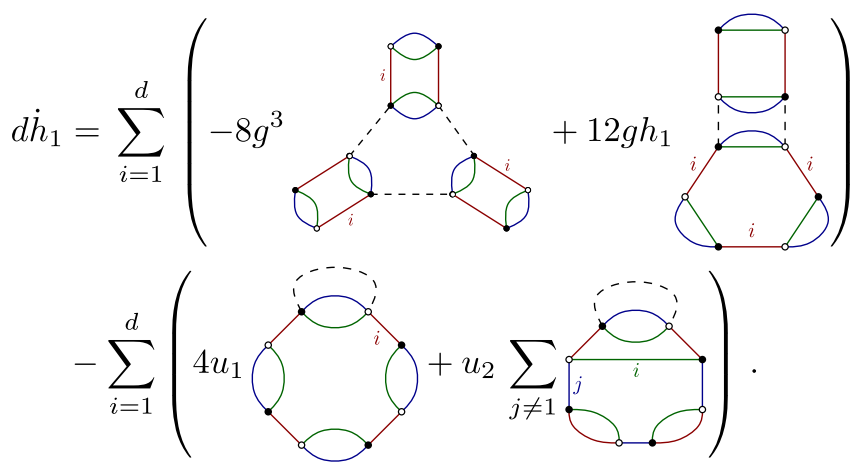

Finally, we get for octic couplings,

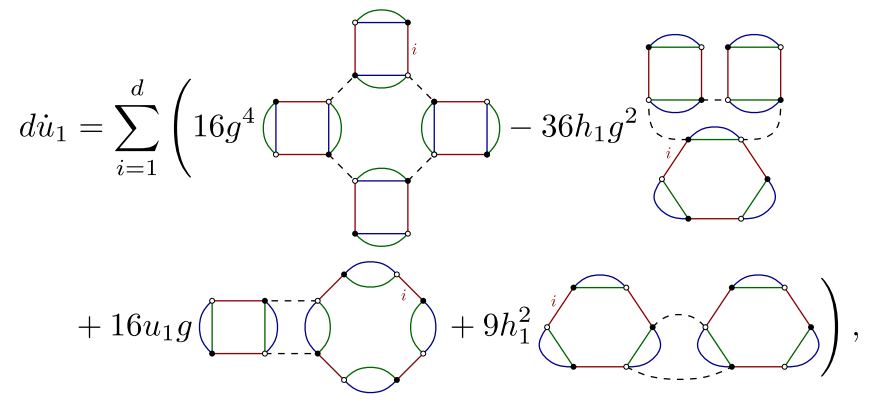

for $u_{1}$,

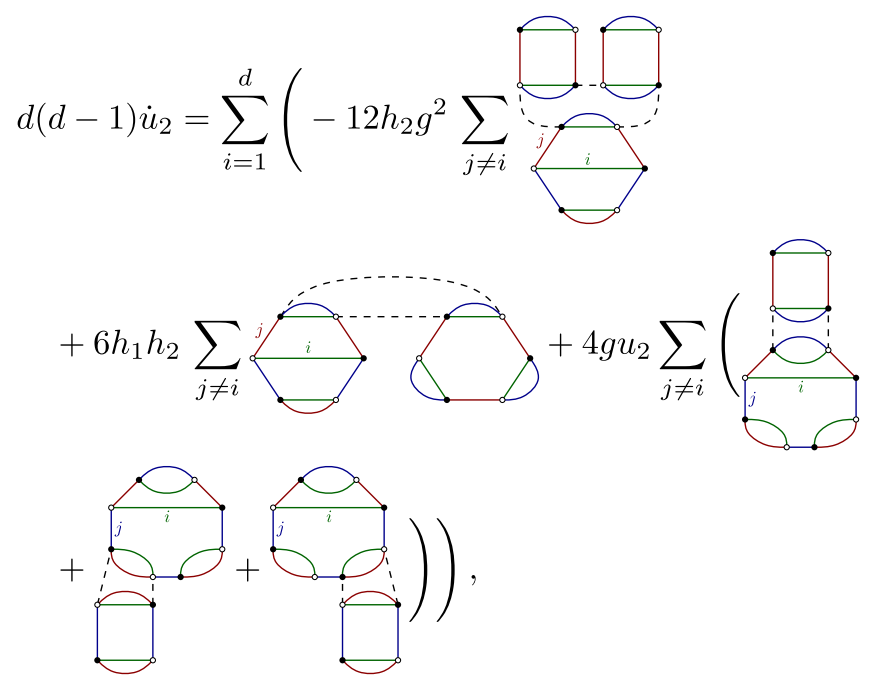

for $u_{2}$,

$$
d(d-1) \dot{u}_{3}=\sum_{i, j \neq i}\left(8 g u_{3}\right.
$$

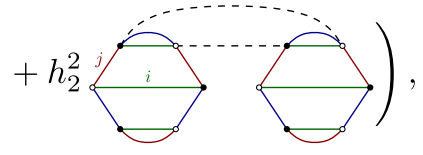

for $u_{3}$,

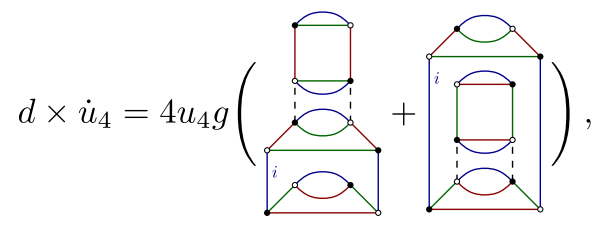

for $u_{4}$, and finally for $u_{5}$, 


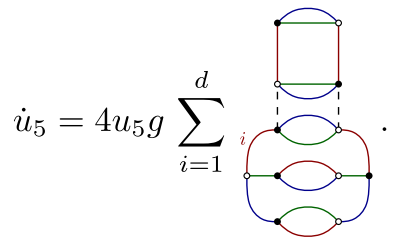

These graphical equations may be easily translated in ordinary equations. Defining the scheme-dependent symbols $L_{n}^{(S)}(\eta)$ as

$L_{n}^{(S)}\left(\eta^{(S)}\right):=\eta^{(S)}\left(\iota_{-1, n}-\imath_{0, n}\right)+l_{-1, n}+(1-\alpha) \partial \iota_{0, n}^{(S)}$,

we get straightforwardly,

$$
\begin{aligned}
\beta_{g}^{(S)}= & 2\left(1-\eta^{(S)}\right) \bar{g}+4 \bar{g}^{2}(k) L_{3}^{(S)}-\left(3 \bar{h}_{1}+2 \bar{h}_{2}\right) L_{2}^{(S)}, \\
\beta_{h_{1}}^{(S)}= & \left(4-3 \eta^{(S)}\right) \bar{h}_{1}-8 \bar{g}^{3} L_{4}^{(S)}+12 \bar{g} \bar{h}_{1} L_{3}^{(S)} \\
& -\left(4 \bar{u}_{1}+2 \bar{u}_{2}\right) L_{2}^{(S)}, \\
\beta_{h_{2}}^{(S)}= & \left(4-3 \eta^{(S)}\right) \bar{h}_{2}+8 \bar{g} \bar{h}_{2} L_{3}^{(S)} \\
& -\left(2 \bar{u}_{2}+\bar{u}_{5}+2 \bar{u}_{4}+4 \bar{u}_{3}\right) L_{2}^{(S)}, \\
\beta_{u_{1}}^{(S)}= & \left(6-4 \eta^{(S)}\right) \bar{u}_{1}+16 \bar{g}^{4} L_{5}^{(S)}-36 \bar{h}_{1} \bar{g}^{2} L_{4}^{(S)} \\
& +\left(16 \bar{u}_{1} g+9 \bar{u}_{1}^{2}\right) L_{3}^{(S)}, \\
\beta_{u_{2}}^{(S)}= & \left(6-4 \eta^{(S)}\right) \bar{u}_{2}-12 \bar{h}_{2} \bar{g}^{2} L_{4}^{(S)}+6 \bar{h}_{1} \bar{h}_{2} L_{3}^{(S)} \\
& +12 \bar{g}_{2} L_{3}^{(S)}, \\
\beta_{u_{3}}^{(S)}= & \left(6-4 \eta^{(S)}\right) \bar{u}_{3}+8 \bar{g} \bar{u}_{3} L_{3}^{(S)}+\bar{h}_{2}^{2} L_{3}^{(S)}, \\
\beta_{u_{4}}^{(S)}= & \left(6-4 \eta^{(S)}\right) \bar{u}_{4}+8 \bar{u}_{4} \bar{g} L_{3}^{(S)}, \\
& \beta_{u_{5}}^{(S)}=\left(6-4 \eta^{(S)}\right) \bar{u}_{5}+12 \bar{u}_{5} \bar{g} L_{3}^{(S)} ;
\end{aligned}
$$

the expression for $\eta^{(S)}$ being unchanged:

$$
\eta^{(S)}:=-6 \bar{g} \frac{l_{-1,2}+(1-\alpha) \partial t_{0,2}^{(S)}}{1+6 \bar{g}\left(l_{-1,2}-l_{0,2}\right)}
$$

Investigating numerically the fixed points, respectively for sextic and octic truncations, we get a very large number of solutions. Some of them are irrelevant, violating the regulator bound $\eta=-1$, which seems to be very unstable passing from sextic to octic truncations. Some of them moreover involve more than one relevant direction, and may be interpreted as multicritical points, corresponding to a triple scaling limit and so one $[102,103]$. Finally, only one fixed point is physically relevant for the double scaling limit, involving only one relevant direction, and has a small dependence on the truncation level. The results for quartic, sextic, and octic interactions are summarized in Table I. Note that at this fixed point, only couplings $g, h_{1}$, and $u_{1}$
TABLE I. Characteristics of the non-Gaussian fixed point relevant for the double-scaling limit for octic melonic truncations.

\begin{tabular}{llccc}
\hline \hline Truncations order & $\alpha$ and scheme & $\bar{g}$ & Relevant $\theta$ & $\eta$ \\
\hline 4 & $\alpha=1$ & -0.04 & 2.39 & 0.80 \\
4 & $\alpha=2\left(S_{1}\right)$ & 0.006 & 3.35 & 0.65 \\
4 & $\alpha=\frac{3}{2}\left(S_{2}\right)$ & 0.016 & 2.26 & 0.57 \\
6 & $\alpha=1$ & -0.035 & 2.34 & 0.71 \\
6 & $\alpha=2\left(S_{1}\right)$ & 0.0046 & 3.04 & 0.50 \\
6 & $\alpha=\frac{3}{2}\left(S_{2}\right)$ & 0.01 & 2.19 & 0.42 \\
8 & $\alpha=1$ & -0.03 & 2.31 & 0.65 \\
8 & $\alpha=2\left(S_{1}\right)$ & 0.004 & 2.85 & 0.40 \\
8 & $\alpha=\frac{3}{2}\left(S_{2}\right)$ & 0.01 & 2.16 & 0.35 \\
\hline \hline
\end{tabular}

take a nonzero value. All other couplings vanish exactly, and the results are essentially insensitive to their presence on the truncation (as we can check explicitly, see the next subsection). This may be viewed as an indication that only a subfamily of melons contributes to the fixed point structure, especially in regard to the understanding of the double scaling limit using renormalization group. This subfamily is known as a nonbranching melonic sector, and nonbranching melons may be defined recursively as pictured in Fig. 8. We will use this observation in the next section to construct truncations up to order 20 in the nonbranching sector. Another interesting observation can be given by the following prescription: one can mention that the range of values for the couplings at the fixed point seems to follow an interesting hierarchy, $h_{1} \sim g / 10^{n}$, $u_{1} \sim h_{1} / 10^{n}, n$ being of order 1 for a standard Litim regulator, and between 1 and 2 for regulators with $\alpha=2$ and $\alpha=3 / 2$, in schemes $S_{1}$ and $S_{2}$ respectively. This shows that no significant interacting structure appears up to order $g$.

From the results summarized in [102,103] Table I, the following essential observations can be made:

(1) First of all, the characteristics of the fixed point are essentially the same between all the regularization schemes. This concerns both the values of the critical exponents and the values of the anomalous dimension.

(2) The values of the relevant characteristics seem to converge toward a finite limit. This is especially the case for the critical exponent, which seems to
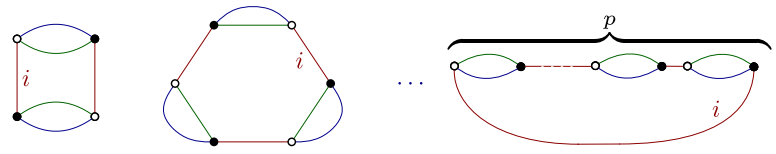

FIG. 8. Structure of the nonbranching melon in rank 3. The last bubble involves $2 p$ nodes along the ring of color $i$. We call 2-dipole the insertions along this mono-colored ring, built as two black and white nodes hooked together by two colored edges of color $\neq i$. 
converge toward 2. The large truncations that we will consider in the next section for the nonbranching sector and the exact results deduced from the EVE method in the next section will confirm this heuristic bound.

(3) Taking Ward identities into account may improve the results qualitatively, compared with the exact results. This is indeed the case for the scheme $S_{2}$, which effectively improves the result, at the orders considered, compared with the results obtained with the Litim regulator. Besides, the rate of convergence seems significantly faster, for the critical exponent than the anomalous dimension. However, the difference seems worse in the diagram $S_{1}$, which however displays a speed of convergence greater than the diagram $S_{1}$ when the order of the truncation increases. Besides, the convergence is much slower using the Litim regulator.

From this third point, we deduce that in the point of view of the proximity to the exact result and the speed of convergence with the order of truncation, it seems that any regularization improving the disagreement with Ward identities will be better than the regular Litim regulator. This result will be supported with large order investigations in the next section. Even to close this part, we aim to add an important remark about the violation of Ward identities. One may object that, even if we fine-tune the regulator to vanish $\mathcal{L}_{2}$, the disagreement from coefficients that are not canceled could be worse. It is, however, easy to check that this is not the case. Indeed, from the computations performed in the previous section [Eq. (49)], we see for instance that using scheme $S_{1}$, the additional factor $2^{p}$ arising, setting $\alpha=2$, is compensated by the fact that, numerically, $\left|\bar{g}_{\text {Litim }}\right|>2 \bar{g}_{S_{1}}$, which becomes the tendency that seems to increase with the order of truncation. The same conclusion occurs for the regularization $S_{2}$. Moreover, the improvement of the hierarchical behavior for higher couplings at the fixed point when $\alpha \neq 1$ enforces this observation.

\section{Nonbranching sector up to order 20}

The nonbranching sector follows a well-know recursive definition, and in this sector, one can easily find an expression for the $\beta$ functions for arbitrary order. For convenience, we introduce the notation $u_{2 q}$ for the renormalized coupling (for instance $u_{4}=g$ and so one). It is therefore easy to check recursively that [60]

$$
\begin{aligned}
\beta_{2 p}^{(S)}= & (2(p-1)-p \eta) u_{2 p}+\sum_{k=1}^{p}(-1)^{k} L_{k+1}^{(S)}(\eta) \\
& \times \sum_{\left\{n_{2 q}\right\} \in \mathcal{D}_{k, p}} \frac{k !}{\prod_{q \geq 2} n_{2 q} !} \prod_{q \geq 2}\left(q u_{2 q}\right)^{n_{2 q}},
\end{aligned}
$$

where $n_{2 q}$ denotes the number of interactions involved on the loop of length $k$, and $\mathcal{D}_{k, p}$ is the set of $\left\{n_{2 q}\right\}$ satisfying the two conditions:

$$
\sum_{q \geq 2} n_{2 q}=k, \quad \sum_{q \geq 2} q n_{2 q}=p+q,
$$

the first constraint being interpreted as the length of the loop equal to $k$, and the second constraint takes into account that we construct an effective coupling of valence $2 p$. Finally, it is easy to count the number of contractions leading to a given nonbranching melonic interaction of valence $2 p$. Each bubble of type $2 q$ involved in a loop has $q$ different positions at the leading order and corresponding to the permutation of the 2 dipoles along the monocolored ring. With $n_{2 q}$ diagrams, this leads to a factor $q^{n_{2 q}}$. Moreover, the $k$ bubbles contributing to the loop of length $k$ can be randomly arranged, for the singular propagator $\dot{r}_{k}\left(G^{(2)}\right)^{2}$ (all the other contractions are involved only in the effective propagator $G^{(2)}$ ). The number of arrangements is given by the generalized binomial coefficients:

$$
\mathcal{C}_{k}^{\left\{n_{2 q}\right\}} \frac{k !}{\prod_{q \geq 2} n_{2 q} !},
$$

and the formula (70) follows.

We investigated numerically truncations up to order 20 in this section, and a first observation is that, for the fixed point relevant for the double scaling limit, the presence of a branching melon has no significant effect on the computation of universal quantities, especially on the values of the critical exponents and anomalous dimension. The results are summarized in Figs. 9 and 10. These figures confirm the assumptions that we have done from octic truncations. In Fig. 9, we show that in all cases the value of the critical

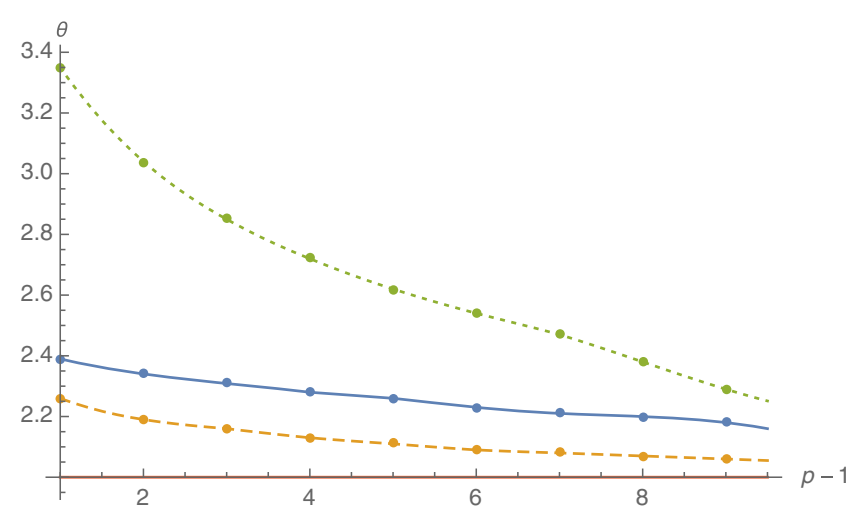

FIG. 9. The relevant critical exponents in the nonbranching sector for truncations up to order 20. The $x$ axis refers to the order of the truncation $p-1$. The blue (solid) curve is for the standard Litim regulator, the green (dotted) curve is for the scheme $S_{1}$ $(\alpha=2)$, and the yellow (dashed) curve is for the scheme $S_{2}(\alpha=3 / 2)$. 


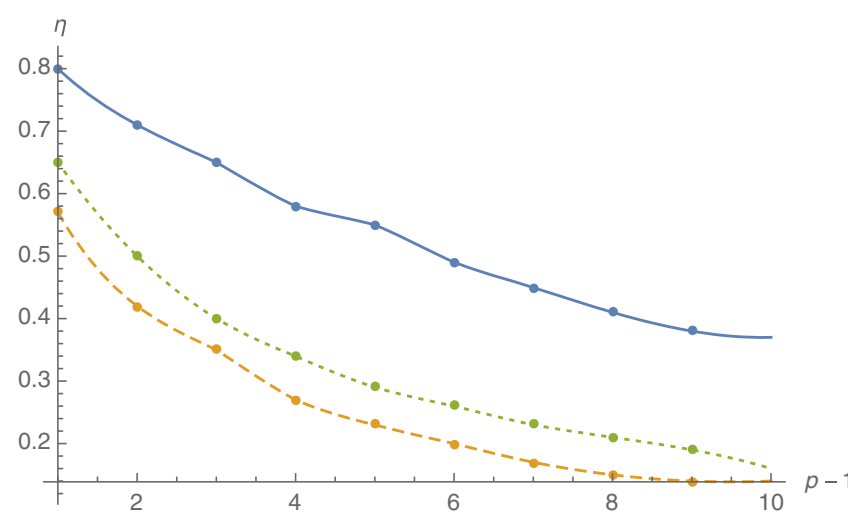

FIG. 10. The corresponding anomalous dimension in the nonbranching sector for truncations up to order 20. Once again, the $x$ axis refers to the order of the truncation $p-1$. Moreover the color conventions are the same as in the previous figure. The blue (solid) curve is for the standard Litim regulator, the green (dotted) curve is for the scheme $S_{1}(\alpha=2)$, and the yellow (dashed) curve is for the scheme $S_{2}(\alpha=3 / 2)$.

exponent is improved by the order of the truncation, going more and less rapidly toward the exact value 1 . However, the observed tendency in octic truncation seems to be confirmed. The values progress in the direction of the $x$ axis but seem to converge toward 2 rather than 1 . Despite this disagreement, this value 2 has a physical meaning. It is nothing but the perturbative result in the first order, and we are tempted to conclude that a purely local truncation cannot significantly improve the physical result, better than the one-loop result. Indeed, the $\beta$ function at one loop must have the following structure:

$$
\beta=(d-1) g+A g^{2},
$$

where $A$ is a constant. This beta function has a fixed point for $g^{*}=-(d-1) / A$, and the critical exponent reads as

$$
\theta_{\text {one-loop }}=-\beta^{\prime}\left(g^{*}\right)=-(d-1)-2 A g^{*}=d-1,
$$

which reduces to 2 for $d=3$. One expects that for a rank $d$ model, the critical exponent will converge toward $d-1$. We will moreover prove in Sec. III E that $\theta$ must be equal to $d-1$ for arbitrary large truncations in the nonbranching sector using the EVE techniques. Then, remembering that our aim is first to evaluate the quality of the truncation, we have to take the bound $\theta=d-1$ as reference. We will see further in the next section that disconnected pieces play an important role if we aim to reach the exact value of $\theta=d-2$.

To summarize Fig. 9, we conclude that, despite a bad start for the critical exponent using scheme $S_{1}$ with small truncations, the speed of convergence is increased when the disagreement with Ward identities is reduced. The best choice seems to be the scheme $S_{2}$, which improves both the rapidity of the convergence and the difference to the inductive limit $d-1$ at each order. However, the scheme
$S_{2}$ could compensate this badly, starting by its record convergence speed. The stability of the results for truncations involving a larger family of graphs could be, ultimately, the only way to decide between the reliability of the two schemes. Figure 10 for the anomalous dimension enforces this conclusion. For the schemes $S_{1}$ and $S_{2}$, the anomalous dimension seems to converge rapidly toward a very small inductive limit, when the progression seems to be slowly using the Litim regulator.

\section{Disconnected pieces}

In the last sections, we considered connected melonic truncations up to valence 8 and a nonbranching sector up to valence 20 . We observed that our results are strongly improved by increasing the order of the truncation, and we expect that this regular progression could converge for sufficiently large truncations. However, we showed that the expected limit does not reach the theoretical result of $\theta=1$, but becomes $\theta=2$. We provide an explanation of this phenomena in the next section. Nevertheless, we completely neglected the influence of next-to-leading order bubbles. One may expect that this can be an important mistake for a theory whose interacting fixed point structure arises from the irrelevant couplings. Indeed, from the power counting (24), we have seen that nonmelonic pieces must have larger canonical dimension than some melonic interactions, and should be included in the truncations. Therefore, for higher-order truncations, one can expect that NLO bubbles could play an important role, especially about the bad melonic limit $\theta=2$. It would not be surprising, moreover, that the NLO contributions play such a role, the double scaling limit being by nature the result which taking into account the influence of the subdominant sectors at the critical point. A complete investigation of the influence of subdominant orders is reserved for the other article. However, the question of the role of disconnected diagrams is expected to be completely different. Indeed, such a diagram arises for instance from the contraction

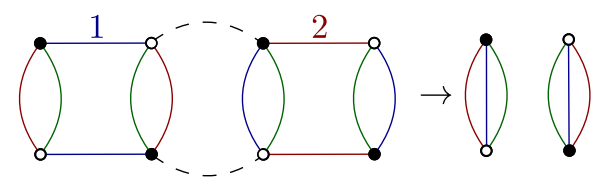

and does not appear for instance in a truncation involving only a single quartic melonic interaction among the $d$ ones. Therefore, we can in principle discard the influence of disconnected pieces from a breaking of the color symmetry invariance of the models considered above. The results are summarized on Table II, using scheme $S_{2}$. We show that the results are significantly closer to the exact limit of $\theta=2$, and, once again, the regulator $\alpha=3 / 2$ is quantitatively better than the standard Litim regulator. Interestingly, the convergence of the anomalous dimension becomes more 
TABLE II. Characteristics of the non-Gaussian fixed point relevant for the double-scaling limit for a single-colored melonic truncation. We considered complete melonic truncations up to order 8 , and only the nonbranching sector up to order 20 .

\begin{tabular}{llccc}
\hline \hline Truncations order & $\alpha$ (scheme $\left.S_{2}\right)$ & $\bar{g}$ & Relevant $\theta \eta$ & \\
\hline 4 & $\alpha=1$ & -0.08 & 2.26 & 0.57 \\
4 & $\alpha=\frac{3}{2}\left(S_{2}\right)$ & 0.036 & 2.13 & 0.31 \\
6 & $\alpha=1$ & -0.06 & 2.18 & 0.21 \\
6 & $\alpha=\frac{3}{2}\left(S_{2}\right)$ & 0.02 & 2.08 & 0.18 \\
8 & $\alpha=1$ & -0.05 & 2.15 & 0.33 \\
8 & $\alpha=\frac{3}{2}\left(S_{2}\right)$ & 0.01 & 2.06 & 0.14 \\
20 & $\alpha=1$ & -0.02 & 2.06 & 0.13 \\
20 & $\alpha=\frac{3}{2}\left(S_{2}\right)$ & 0.005 & 2.02 & 0.04 \\
\hline \hline
\end{tabular}

precise towards the value 0 , and by using the regulator $\alpha=3 / 2$ (see claim 1 of the next section).

As firstly pointed out in [104], the influence of disconnected interactions is not to improve the precision of the critical exponent in regard to the double scaling limit, but to create new fixed points having more than one relevant direction. Such a fixed point is interpreted by the authors as an evidence for a scaling limit beyond double scaling, providing a new continuum limit. However, recovering the double scaling seems to require a specific phase space parametrization, breaking the color symmetry. Indeed, we showed that our results in the previous section, taking into account only the melonic sector, is in agreement with the simplest truncation breaking the color symmetry, but, rigorously, we have no reason to discard the disconnected pieces in the color-symmetric truncations, and the relevant fixed point for double scaling disappears. This pathology has been pointed out as a consequence of finite truncations in [104].

From the power counting (24), an interaction of the form

$$
\left(\begin{array}{l}
\infty \\
\infty
\end{array}\right)
$$

has canonical dimension -3 . In contrast, the valence 6 melonic bubbles have dimension $-2(d-1)=-4$. Therefore, from a strict power counting point of view, there is no reason to discard disconnected pieces. This also concerns the case for the disconnected piece:

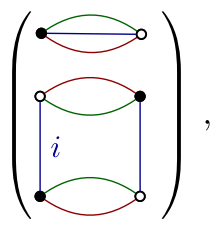

which has power counting dimension -5 , smaller than the dimension of melonic octic truncations, which is -6 . In this section, we briefly consider their influence. Let us consider the colored symmetric truncation:

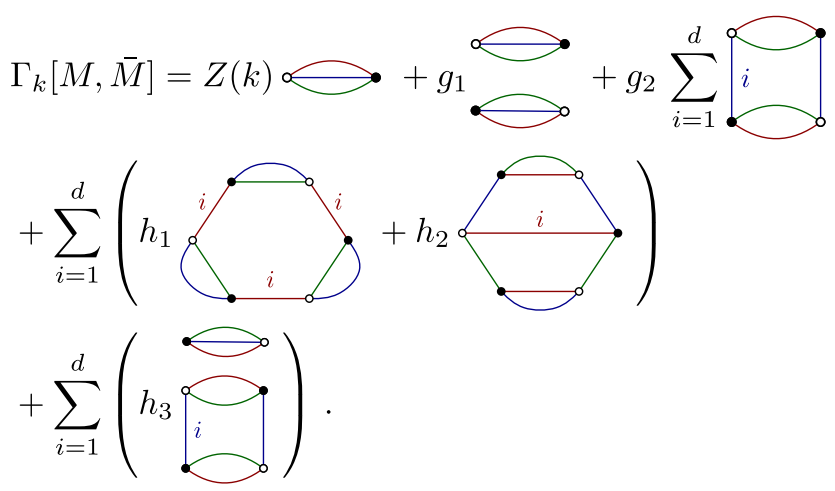

The disconnected terms do not affect the flow equations for $h_{1}$ and $h_{2}$ computed in the previous section (setting to zero the octic couplings). The flow equation for $g_{2}$ receives the additional contribution

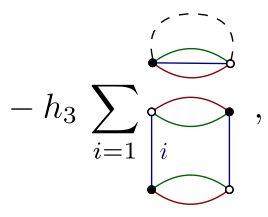

and the expression of $\dot{Z}$ becomes

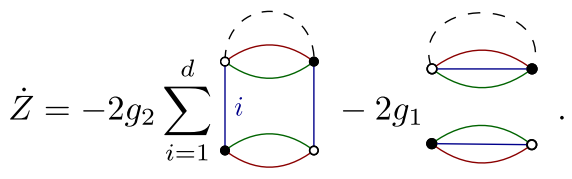

Moreover, the couplings $g_{1}$ and $h_{3}$ have their own flow equations, explicitly,

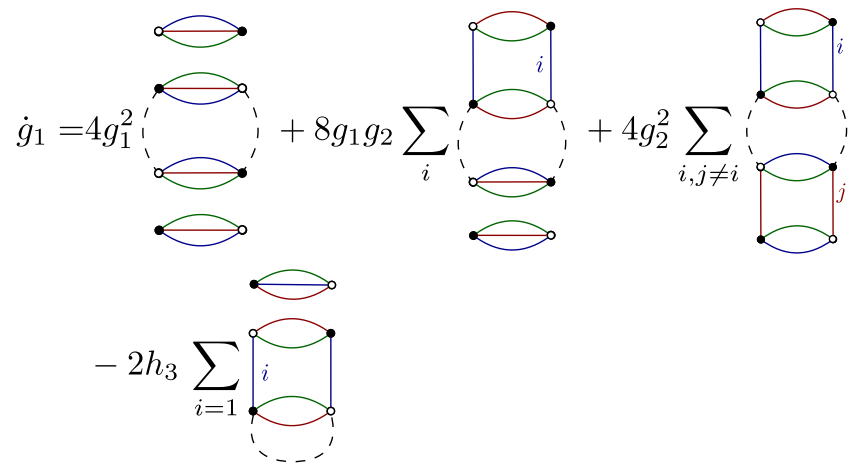

and 


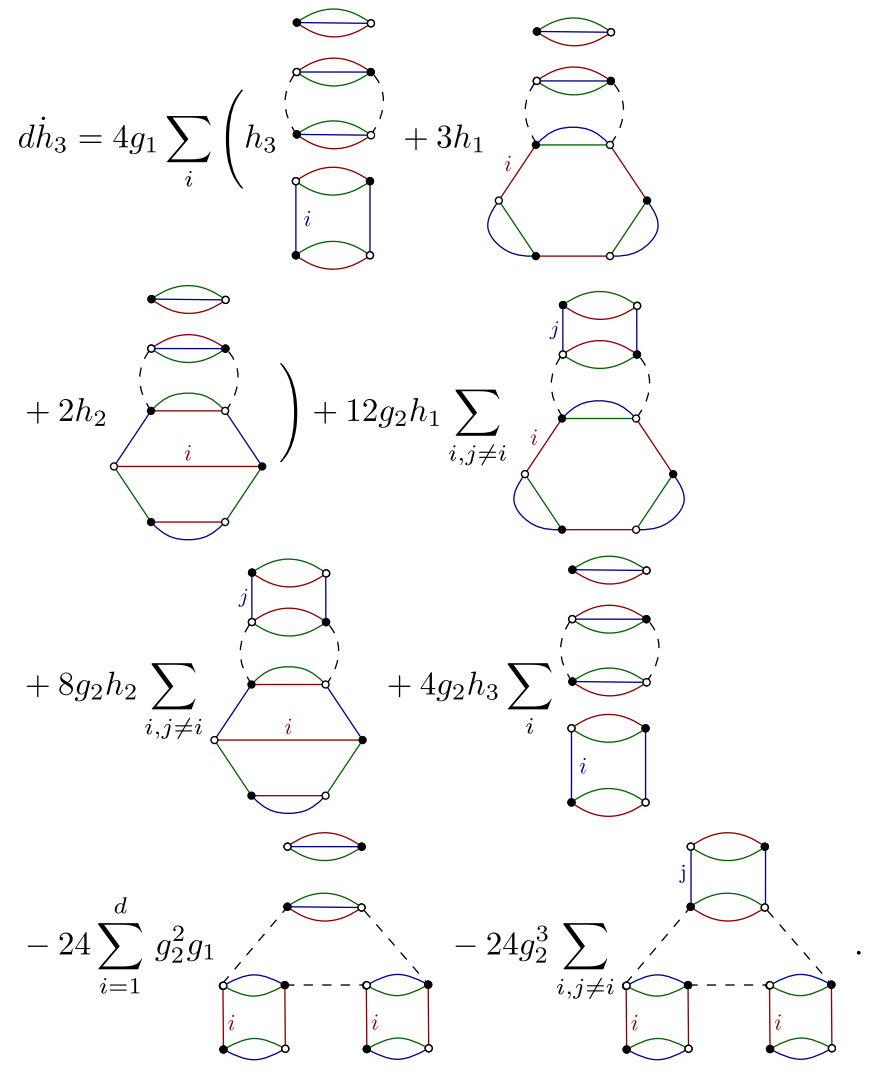

This leads to the following system:

$$
\begin{aligned}
\beta_{g_{1}}^{(S)}= & \left(3-2 \eta^{(S)}\right) \bar{g}_{1}-6 \bar{h}_{3} L_{2}^{(S)}+4 \bar{g}_{1}^{2} K_{3}^{(S)}+24 \bar{g}_{1} \bar{g}_{2} L_{3}^{(S)} \\
& +24 \bar{g}_{2}^{2} J_{3}^{(S)}, \\
\beta_{g_{2}}^{(S)}= & 2\left(1-\eta^{(S)}\right) \bar{g}_{2}+4 \bar{g}_{2}^{2} L_{3}^{(S)}-\left(3 \bar{h}_{1}+2 \bar{h}_{2}\right) L_{2}^{(S)}-\bar{h}_{3} K_{2}^{(S)} \\
\beta_{h_{1}}^{(S)}= & \left(4-3 \eta^{(S)}\right) \bar{h}_{1}-8 \bar{g}_{2}^{3} L_{4}^{(S)}+12 \bar{g}_{2} \bar{h}_{1} L_{3}^{(S)} \\
\beta_{h_{2}}^{(S)}= & \left(4-3 \eta^{(S)}\right) \bar{h}_{2}+8 \bar{g} \bar{h}_{2} L_{3}^{(S)}, \\
\beta_{h_{3}}^{(S)}= & \left(5-3 \eta^{(S)}\right) \bar{h}_{3}+4 \bar{g}_{1} \bar{h}_{3} K_{3}^{(S)}+12 \bar{g}_{1} \bar{h}_{1} L_{3}^{(S)} \\
& +8 \bar{g}_{1} \bar{h}_{2} L_{3}^{(S)}-24 \bar{g}_{2}^{2} \bar{g}_{1} L_{4}^{(S)}-48 \bar{g}_{2}^{3} J_{4}^{(S)} \\
& +16 \bar{g}_{2} \bar{h}_{2} J_{3}^{(S)}+4 \bar{g}_{2} \bar{h}_{3} K_{3}^{(S)} .
\end{aligned}
$$

In these equations we introduced $K_{n}^{(S)}$ defined as

$$
K_{n}^{(S)}:=\frac{Z^{n-1}}{k^{2}} \int d^{d} x \frac{\dot{r}_{k}(x)}{\left(Z+r_{k}(x)\right)^{n}}=: K_{n}^{(1, S)} \eta^{(S)}+K_{n}^{(2, S)},
$$

and the anomalous dimension $\eta^{(S)}$ is given by

$$
\eta^{(S)}=-\frac{6 \bar{g}_{2} L_{2}^{(2, S)}+2 \bar{g}_{1} K_{2}^{(2, S)}}{1+6 \bar{g}_{2} L_{2}^{(1, S)}+2 \bar{g}_{1} K_{2}^{(1, S)}},
$$

where $K_{n}^{(1)}$ and $K_{n}^{(2)}$ can be computed exactly as (see Appendix)

$$
K_{n}^{(1)}=\frac{d^{3}}{2}\left(\frac{1}{n+2}-\frac{\alpha}{n+3}\right) \alpha^{n+2}
$$

and

$$
K_{n}^{(2)}=\frac{d^{3}}{2} \frac{1}{n+2} \alpha^{n+2}+\frac{d^{3}}{2}(1-\alpha) \alpha^{n+2} .
$$

We moreover introduced the one-dimensional integrals:

$J_{n}^{(S)}:=\frac{Z^{n-1}}{k^{2}} \int d x \frac{\dot{r}_{k}(x)}{\left(Z+r_{k}(x)\right)^{n}}=: J_{n}^{(1, S)} \eta^{(S)}+J_{n}^{(2, S)}$.

Explicitly,

$$
J_{n}^{(1, S)}=d\left(\frac{1}{n}-\frac{\alpha}{n+1}\right) \alpha^{n},
$$

and

$$
J_{n}^{(2, S)}=d\left(\frac{1}{n}+(1-\alpha)\right) \alpha^{n} .
$$

Note that it is clear that fixed points discarding the disconnected pieces cannot be a fixed point of the previous system. This can be easily checked at the lowest order, keeping only the quartic disconnected pieces (the coupling $\left.g_{1}\right)$. Setting $g_{1}=0$, the corresponding flow equation involves the product $d(d-1) g_{2}^{2}$, which does not vanish, except for $g_{2}=0$ or if we consider only one quartic melon among the $d$ allowed (i.e., if we break the color permutation symmetry).

We work only with the scheme $S_{2}$, and consider the values $\alpha=1$ and $\alpha=3 / 2$. Starting with the quartic truncation, we get a large number of isolated fixed points. Some of them, however, have to be discarded, violating the regulator bound $\eta=-1$, or being below the singularity line defined by the denominator of $\eta{ }^{6}$ For $\alpha=1$, the physical relevant fixed point closer to the Gaussian fixed point vanishes the branching couplings $\left(\bar{h}_{2}=0\right)$, and the critical exponents take the values

$$
\Theta_{\alpha=1}=(5.22,0.52,-2.40,-1.49),
$$

with anomalous dimension $\eta_{\alpha=1} \approx 1.14$. For $\alpha=3 / 2$, we recover a fixed point reminiscent of this one for values

$$
\Theta_{\alpha=3 / 2}=(8.99,1.64,-6.84,-2.79),
$$

\footnotetext{
${ }^{6}$ The singularity line defined by the denominator of $\eta$ split the phase space in two connected regions. The denominator is moreover positive only in the region connected to the Gaussian fixed point.
} 
and the anomalous dimension $\eta_{\alpha=3 / 2} \approx 1.44$. Note that in the absence of an exact result, we cannot identify which of these results is qualitatively better. The only indication in favor of the second regularization is its good convergence properties in the melonic sector. ${ }^{7}$ Moreover, we show explicitly the disappearance of the fixed point with one relevant direction discovered above, illustrating how the results are strongly dependent on the phase space parametrization.

Now, let us consider the full sextic truncation. Once again, we get a large number of isolated fixed points, but only one of them has stable characteristics. We do not recover the fixed points discovered above, but a fixed point having one relevant complex direction. For $\alpha=1$, we get a nonbranching fixed point having a critical exponent:

$\Theta_{\alpha=1}=(1.48+0.84 i, 1.48-0.84 i,-4.46,-1.59,-1.30)$,

and anomalous dimension $\eta_{\alpha=1} \approx 0.56$. For $\alpha=3 / 2$ we get

$\Theta_{\alpha=3 / 2}=(2.28+0.68 i, 2.28-0.68 i,-4.31,-1.95,-1.56)$,

with anomalous dimension $\eta_{\alpha=3 / 2}=0.33$. Once again, we have no reference to compare these results. However, the characteristics of the fixed points obtained from quartic and sextic truncations seem to be very different. Therefore, a deeper analysis, involving larger truncations, is required to conclude about the reliability of this fixed point, or, as for the melonic sector, a deeper understanding of the exact relations between disconnected pieces, as there exist between connected melonic pieces (see the next section).

\section{E. A limit for the ultralocal melonic approximation}

The previous result showed that the convergence for higher truncation seems to be very dependent on the sectors of the theory space that we take into account. For instance, we showed that taking only the nonbranching melonic sector, for instance, cannot allow reaching the exact value of $\theta=d-2$. This result was in large part empirical because we only considered three regulators among an infinity of possibilities. In this section we provide a solid argument, based on the effective vertex expansion (EVE), showing that even with a truncation of arbitrarily large size, and without making an explicit choice for the regulator, the critical exponent reaches the value $\theta_{\mathrm{op}}=d-1$, which is nothing but the inductive bound discovered from large truncations in the nonbranching sector.

\footnotetext{
${ }^{7}$ Another indication could be the range of values for the couplings at fixed points, sensitively larger for the Litim regulator.
}

Note that in the point of view developed in this paper, the disagreement between the exact value $\theta_{\text {exact }}=d-2$ and $\theta_{\mathrm{op}}$ is not a consequence of the method but of the restricted domain of the full phase space that we investigated. We separate the question of exploring the vast expanses of phase space from the effectiveness of the method, for which we have retained essentially two criteria, namely proximity with an optimal result (in this case $\theta_{\mathrm{op}}=d-1$ ) and the speed of convergence. It is expected that the methods giving good results for specific sectors will be as effective on larger domains, and more likely to allow one to discover new critical behaviors.

The EVE is a recent development for tensorial group field theories [78-86]. It allows the capturing of entire sectors, i.e., an infinite set of effective vertices and their exact momentum dependence, in contrast with crude truncations discussed in the previous section. This method is easy to use only in the nonbranching melonic sector, and we only focus on it in this paper. Extensions to subleading order is a very fastidious task discussed in [79], and no version exists for disconnected interactions. However, the fact that we may be able to keep the complete momentum dependence of the effective vertices could strongly improve the local truncation with the Ward identity violation, without requiring a fine-tuning adjustment. We do not discuss in full detail this issue here, deferring a more exhaustive analysis to a future article. In Sec. V, we will discuss the influence of derivative coupling, and we will return briefly to the EVE at this time.

Let us consider the quartic model described by the classical action:

$$
S(T, \bar{T})=0+g \sum_{i=1}^{\nu} \longrightarrow_{i}^{\infty}
$$

Note that we stopped the sum over melonic interaction to the number $1 \leq \nu \leq d$. The reader may be surprised by this restriction. To be more clear we had not made an explicit choice of classic action before. We implicitly use the same argument of universality [105], arguing that the critical behavior of the tensor models must be the same as for the quartic model. We therefore do not lose anthing by restricting ourselves to a quartic model, with which it is easier to work. Investigating the properties of the leading order (i.e., melonics) diagrams, it is not hard to prove the following statement $[78,79]$ :

Proposition 1. Let $G$ be a nonvacuum 1 PI diagram with $2 n$ external edges. The following properties hold:

(i) The $2 n$ external edges are pairwise connected to $(d-1)$ dipoles. They build $(d-1) n$ open cycles of type $0 i$.

(ii) In addition there exist $n$ open cycles of the same color hooked to external edges pairwise. 


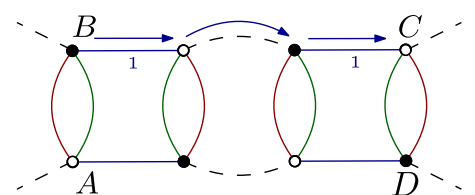

FIG. 11. A melonic 4-point graph, with external nodes labeled as $A, B, C$, and $D$. Pairs $(A, B)$ and $(C, D)$ build $(d-1)$ dipoles. Moreover, pairs $(A, C)$ and $(B, D)$ are boundaries of external cycles of the same colors, one per pair. The corresponding cycle for the pair $(A, C)$ is materialized by the blue arrows.

Figure 11 provides an illustration of this statement, and we recall the definition of a $k$ dipole:

Definition 5. A $k$ dipole is built as two black and white nodes linked together by $k$ colored edges of colors different from 0.

As a direct consequence of this proposition, the Feynman graphs involved in the expansion of the effective vertex functions $\Gamma_{k}^{(2 n)}$ can be labeled by an index $i$ corresponding to the color of the $n$ open cycles. Thus, $\Gamma_{k}^{(2 n)}$ decomposes as a sum of $d$ functions:

$$
\Gamma_{k ; \vec{n}_{1}, \ldots, \vec{n}_{2} n}^{(2 n)}=\sum_{i=1}^{d} \Gamma_{k ; \vec{n}_{1}, \ldots, \vec{n}_{2} n}^{(2 n, i)}
$$

The Feynman diagrams involved in the expansion of $\Gamma_{k ; \vec{n}_{1}, \ldots, \vec{n}_{2} n}^{(2 n, i)}$ fix completely the relation between the different indices. For $n=2$, the relation between the different indices has been described in (10). Graphically,

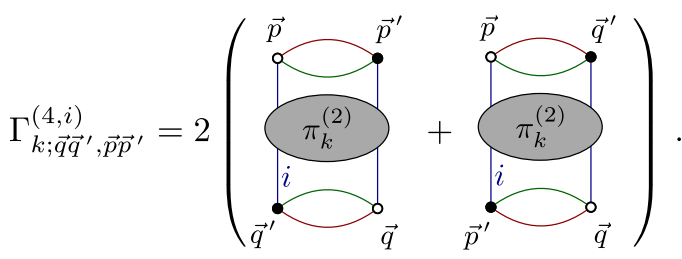

The aim of the EVE is to close the infinite hierarchical system obtained by expanding the exact flow equation (1). Restricting firstly our attention to local couplings, this closure requires one to express the 6-point function $\Gamma_{k ; \vec{n}_{1}, \ldots, \vec{n}_{6}}^{(6, i)}$ in terms of the 4- and 2-point functions. From proposition III E; the 6-points vertex function must have the following structure:

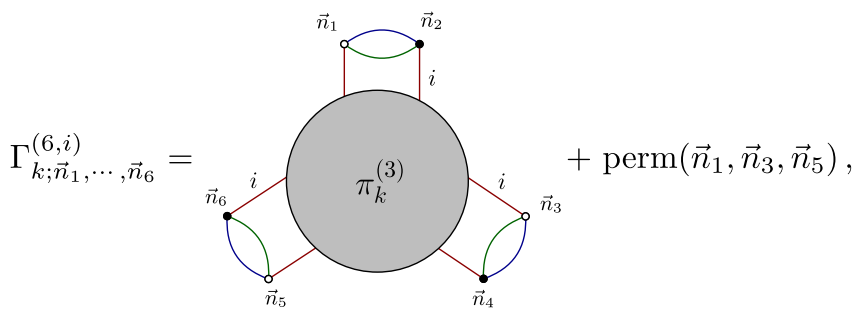

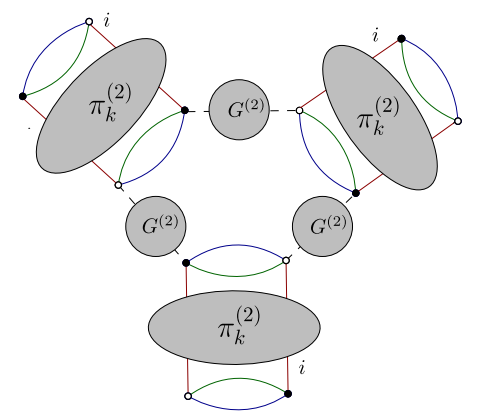

FIG. 12. Internal structure of the 1PI 6-points graphs.

where perm denotes the permutations of the three elements. Denoting formally by $\pi_{k}^{(3)}\left(n_{11}, n_{31}, n_{51}\right)$ the sum of the interiors of the graphs contribute to the perturbative expansion of $\Gamma_{k ; \vec{n}_{1}, \ldots, \vec{n}_{6}}^{(6, i)}$. The explicit expressions of $\pi_{k}^{(2)}$ and $\pi_{k}^{(3)}$ can be easily obtained from the recursive structure of melonic diagrams [81]. For $\pi_{k}^{(2)}$, we get

$$
\begin{aligned}
\pi_{k}^{(2)}(n, n) & =g\left(1-2 g \mathcal{A}_{2, n}+4 g^{2}\left(\mathcal{A}_{2, n}\right)^{2}+\cdots\right) \\
& =\frac{g}{1+2 g \mathcal{A}_{2, n}},
\end{aligned}
$$

where

$$
\mathcal{A}_{m, n}:=\sum_{\vec{n}}\left(g^{(2)}(\vec{n})\right)^{m} \delta_{n_{1} n} .
$$

In the same way, the internal structure of the 6-point melonic diagram can be investigated recursively. The explicit structure is given in Fig. 12, which can be translated as

$$
\pi_{k}^{(3)}(n, n, n)=2\left(2 \pi_{k}^{(2)}(n, n)\right)^{3} \mathcal{A}_{3, n} ;
$$

the combinatorial factor 2 in front of $\pi_{k}^{(2)}$ arises from the two allowed orientations for the boundary effective 2 -points vertices. Following [84], we call structure equations the relations (100), (96), and (98) between effective melonic vertices. Note that, even if we focus on the first relations, such a relation exists to all orders, and the $2 n$ point functions may be expressed in terms of the 4- and 2-point functions ${ }^{8}$ [81]. Interestingly, all the effective vertices depend on the knowledge of the 2-point function. This function, or more precisely the self-energy $\Sigma_{k}(\vec{n})$ is determined in the melonic sector from a closed equation. Like the structure equations (96) and (98), the closed equation arises directly from proposition III $\mathrm{E}$, and the reader may consult $[48,106]$ and references therein. Defining the monocolored 2-point functions $\sigma_{k}$ as

\footnotetext{
${ }^{8}$ Note that these structure equations are nothing but the melonic version of the well-known Schwinger-Dyson equations.
} 


$$
\Sigma_{k}(\vec{n}):=\sum_{i=1}^{d} \sigma_{k}\left(n_{i}\right)
$$

which is nothing but the transcription of Eq. (93) for 2-point functions, we have the following statement:

$$
\sigma_{k}(n)=-2 g \sum_{\vec{n}} \delta_{n_{1} n} \frac{1}{1-\sum_{i=1}^{\nu} \sigma_{k}\left(n_{i}\right)+r_{k}(\vec{n})} .
$$

We will use this equation especially in Sec. V, investigating the momentum dependence of the melonic functions, in regard to modified Ward identities. Expanding the flow equation (1) and keeping only the leading-order terms in the large- $k$ limit, we get, using the same notations as in the previous section:

$$
\dot{\gamma}_{k}^{(2)}(\vec{n})=-\sum_{i=1}^{d} \underbrace{\pi_{k}^{(2)}}_{\vec{n}}
$$

and

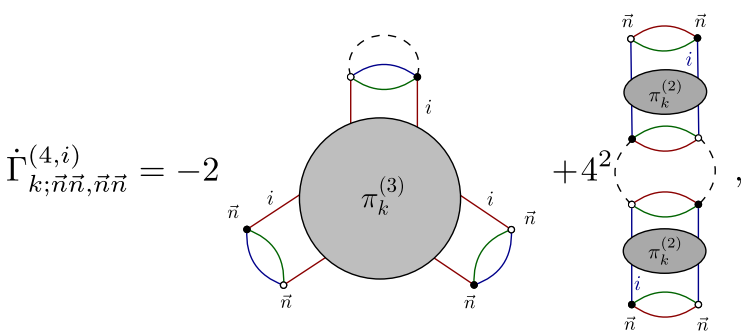

the factor 2 in front of the six points contribution arising from the remaining permutation of external edges hooked to white nodes. In this form, the RG equations are completely closed, the 6-point function being expressed in terms of 2- and 4-points ones. Setting the external momenta to zero, and from the renormalization condition

$$
\pi_{k}^{(2)}(0,0) \equiv g(k)
$$

we deduce

$$
\eta^{(S)}=-2 \nu \bar{g}(k) L_{2}^{(S)}(\eta),
$$

and

$$
\begin{aligned}
\beta_{g, E V E}= & ((d-1)-2 \eta) \bar{g}(k)+4 \bar{g}^{2}(k) L_{3}(\eta) \\
& -\frac{1}{2} \pi_{k}^{(3)}(0,0,0) L_{2}(\eta),
\end{aligned}
$$

where in (104) $\nu$ is equal to the number of quartic melonic interactions that we have in the classical action (92). These equations are exact, in the sense that they do not require more than exact relations between melonic observables at leading order. At this stage, we can address the following issue: Assuming that we intend to construct an ultralocal approximation of the effective action from an arbitrarily wide truncation, such that this approximation is compatible with the constraints given by the EVE, the $\beta$ function, in an ultralocal truncation, has been computed in the previous section, and it corresponds to the previous equation, taking into account the definition $(3 !)^{2} h_{1}=3 ! \pi_{k}^{(3)}(0,0,0)$. However, there is another constraint, arising from the definition (103) of the effective coupling. Indeed, taking the first derivative with respect to $k$, we get, using (96)

$\dot{g}(k)=4 g^{2}(k) \sum_{\vec{n}} \delta_{n_{1} 0} \frac{-\sum_{i} \frac{d \sigma_{k}}{d t}\left(n_{i}\right)+\frac{d r_{k}}{d n}(\vec{n})}{\left(1-\sum_{i=1}^{\nu} \sigma\left(n_{i}\right)+r_{k}(\vec{n})\right)^{3}}$.

Recognizing that $\frac{d \sigma_{k}}{d t}$ is nothing but $-\eta / \nu$ in local approximation, we thus obtain

$\beta_{g}^{\text {(Exact) }}=((d-1)-2 \eta) \bar{g}+4 \bar{g}^{2} L_{3}(\eta)+4 \bar{g}^{2} \eta \bar{A}_{3,0}$,

where $\bar{A}_{n, 0}$ is the renormalized version of $A_{n, 0}$, extracted by the global $k$ and $Z$ dependence. Note that these equations only depend on the coupling $g(k)$, which is the only one relevant to drive the RG flow (see [79,81] for more details). Equation (107) has to be compared with Eq. (105). From the definition of $\pi_{k}^{(3)}(0,0,0)$ [Eq. (98)], we conclude that the two equations are compatible, and the ultralocal melonic approximation makes sense, if

$$
\bar{A}_{3,0}\left(\eta+2 \bar{g} L_{2}\right)=0,
$$

which has two solutions:

(1) $\bar{A}_{3,0}=0$, and

(2) $\eta=-2 \bar{g} L_{2}$.

We will investigate separately these two conditions. Note that the second one is in conflict with (104) if $\nu \neq 1$. Therefore, if the second condition holds, we have two possibilities: $\nu=1$ or $\nu \neq 1 \Rightarrow \eta=0$. From the second condition, we deduce that Eq. (105) reduces to

$$
\beta_{g}=(d-1) g+4 g^{2} L_{3}(\eta=0),
$$

and, from our perturbative analysis of the previous section [Eq. (73)] admits a non-Gaussian fixed point for $g^{*}=$ $-(d-1) / L_{3}(0)$, with critical exponent,

$$
\theta=-\beta_{g}^{\prime}\left(g=g^{*}\right)=d-1 .
$$

The case $\nu=1$ may be analyzed with more attention. Explicitly, the flow equation for the coupling $g$ reads as 


$$
\beta_{g}=((d-1)-2 \eta) \bar{g}+4 \bar{g}^{2}\left(L_{3}+\eta \bar{A}_{3,0}\right) .
$$

In a purely local approximation, $L_{3}(\eta)$ is given by Eq. (66). To compute $A_{3,0}$, we assume that we work with a sharp regulator ( $f$ is proportional to a Heaviside distributions), so that we have

$$
\bar{A}_{n, 0}=\sum_{\vec{n}} \delta_{n_{1} 0}\left[\frac{\theta\left(\alpha k-\sum_{i} n_{i}\right)}{(1+f(\vec{n}))^{n}}+\theta\left(\sum_{i} n_{i}-\alpha k\right)\right] .
$$

The $f$-dependent part of this equation can be easily computed, and we introduce the notation

$$
\mathcal{S}_{n}:=\sum_{\vec{n}} \delta_{n_{1} 0} \frac{\theta\left(\alpha k-\sum_{i} n_{i}\right)}{(1+f(\vec{n}))^{n}} .
$$

Note that the $f$-independent contribution is $n$ independent as well. Therefore $\bar{A}_{n, 0}-\bar{A}_{m, 0}=\mathcal{S}_{n}-\mathcal{S}_{m}$ is finite. Moreover, from (96), we have

$$
A_{2,0}=\frac{1}{2}\left(\frac{1}{g(k)}-\frac{1}{g(\Lambda)}\right)
$$

for some UV cutoff $\Lambda[g(\Lambda)$ being what we denoted by $g$ in Eq. (96) for instance, i.e., the initial bare coupling]. As a result, we get, after some algebraic manipulations:

$$
\bar{A}_{3,0}=\mathcal{S}_{3}-\mathcal{S}_{2}+\frac{1}{2}\left(\frac{1}{\bar{g}(k)}-\frac{Z^{2}}{k^{2} g(\Lambda)}\right) .
$$

Moreover, following (66),

$$
L_{k}(\eta)=\eta L_{k}^{(1)}+L_{k}^{(2)}
$$

where the $L_{k}^{(j)}$ are independent of $\eta$; it is easy to check, from definition (66) that $\mathcal{S}_{n}-\mathcal{S}_{n-1}=-L_{n}^{(1)}$. Therefore,

$$
L_{3}+\eta \bar{A}_{3,0}=L_{3}^{(2)}+\frac{1}{2}\left(\frac{1}{\bar{g}(k)}-\frac{Z^{2}}{k^{2} g(\Lambda)}\right),
$$

and the $\beta$-function $\beta_{g}$ becomes

$$
\beta_{g}=(d-1) \bar{g}+4 \bar{g}^{2} L_{3}^{(2)}+\bar{g}^{2} \frac{2 \eta Z^{2}}{k^{2} g(\Lambda)} .
$$

This is a nontractable equation, depending on the initial conditions. For $\eta=0$, the difficulty to think about this equation disappears, and we recover the precedent result, with $\theta=d-1$. However, it has two simpler and interesting limit cases. In the deep UV limit $k \approx \Lambda$, we must have

$$
\bar{g}^{2} \frac{2 \eta Z^{2}}{k^{2} g(\Lambda)} \approx 2 \eta \bar{g}
$$

so that the $\beta$ function reduces to

$$
\beta_{g}=((d-1)-2 \eta) \bar{g}+4 \bar{g}^{2} L_{3}^{(2)} .
$$

In contrast, let us consider the intermediate regime $\Lambda \gg k \gg 1$, far from the deep UV regime, but also far enough from the deep IR so that nonmelonic terms are neglected. Remembering that from the power counting $g(\Lambda) \sim \Lambda^{-2}$, we deduce that the $\eta$-dependent term dominates the flow, and

$$
\beta_{g} \approx \bar{g}^{2} \frac{2 \eta Z^{2}}{k^{2} g(\Lambda)}
$$

Equation (121) vanishes only for $\bar{g}=0$. However, the first equation (120) has a more interesting fixed point structure. Solving the equation $\eta=-2 \bar{g}\left(\eta L_{2}^{(1)}+L_{2}^{(2)}\right)$ as

$$
\eta=\frac{-2 \bar{g} L_{2}^{(2)}}{1+2 \bar{g} L_{2}^{(1)}}
$$

we get for $\beta_{g}=0$ the condition

$$
\left((d-1)\left(1+2 \bar{g} L_{2}^{(1)}\right)+4 \bar{g} L_{2}^{(2)}\right)+4 \bar{g}\left(1+2 \bar{g} L_{2}^{(1)}\right) L_{3}^{(2)}=0
$$

where we assumed $\bar{g} \neq 0$. Numerical investigations, using the regulators used in this section, show that the resulting fixed point match with the results of the previous section. In rank 3, we recover a fixed point having essentially the same characteristics as the fixed point obtained from a quartic truncation. More interestingly is the behavior of this solution with the rank $d$ of the tensor. In Fig. III E, we show the behavior of the critical exponent with the rank using the scheme $S_{2}$, and we show that $\theta \geq d-1$, and converge weakly toward this limit.

Now, let us consider the first condition $A_{3,0}=0$. From Eq. (115),

$$
-L_{3}^{(1)}+\frac{1}{2}\left(\frac{1}{\bar{g}(k)}-\frac{Z^{2}}{k^{2} g(\Lambda)}\right)=0 .
$$

Noting that $L_{3}^{(1)}$ is a pure number, this equation can be translated locally as a differential equation:

$$
\dot{\bar{g}}=((d-1)-2 \eta)\left(\frac{Z^{2}}{g(\Lambda) k^{2}}\right) \bar{g}^{2} .
$$

In the deep UV regime, it may be approximated by the most suggesting expression:

$$
\dot{\bar{g}} \approx((d-1)-2 \eta) \bar{g}
$$


The $\beta$ function behaves as if there are no interactions at all. The only trace of the non-Gaussian measure is in the definition of the anomalous dimension and for this reason we refer to this solution as the purely scaling limit. In addition to the Gaussian fixed point, we get the condition

$$
\eta^{*}=\frac{d-1}{2} \Rightarrow \bar{g}^{*}=-\frac{1}{2} \frac{d-1}{2 d L_{2}^{(2)}+(d-1) L_{2}^{(1)}},
$$

leading to the critical exponent

$$
\theta=+\left.2 \frac{\partial \eta}{\partial \bar{g}}\right|_{\bar{g}=\bar{g}^{*}}=(d-1)\left[1+\frac{L_{2}^{(1)}}{L_{2}^{(2)}} \frac{d-1}{2 d}\right] .
$$

Once again, the numerical investigations based on the regulator considered in this paper show that this critical exponent is always bigger than $d-1$. Moreover, we showed that, except for the solution $\eta=0$, all the solutions of (108) do not allow one to obtain autonomous local systems, without dependence on the initial conditions. To summarize, we note the following:

Claim 1. In the UV regime, the compatibility with the melonic structure equations imposes that for any full ultralocal approximation of the effective action, involving only connected bubbles we must have $\eta=0$. Moreover, for the complete truncation, when all the graphs are taken into account, we get $\theta_{\mathrm{op}}=d-1$.

This conclusions are obviously in accordance with our results of the previous subsections. In particular, we showed that any truncation which reduces the Ward identity violation, and therefore improves the reliability of the purely local truncation improves as well the rate of convergence toward the limit $\theta_{\mathrm{op}}=d-1$. In the next subsection, we will consider the effect of disconnected pieces, from a "dressed" parametrization of the local theory space.

To close this section, let us add another important remark. From the structure equations between $2 n$, 4 - and 2-point observables, we were able to close the infinite hierarchy of flow equations in the melonic sector. In this sense, these equations take into account the whole melonic sector. Interestingly, we do not find more than one, or eventually two interacting fixed points. This strongly contrasts with the results obtained in the melonic sector in the previous sections, using large local truncations, where a large number of fixed points were found. Some of these fixed points were interpreted as an artifact of the truncation, but a certain number of them, with more than one relevant direction, seems to stay in high truncations. We see now that these fixed points are artifacts of the truncation as well, which does not take into account the strong relation coming from the structural equations. This appears to be a new effect of the pathology which can appear when the constraints inherent in a given sector are ignored, making all the more difficult confidence in the results coming from a local truncation.
Finally, the reader may be wondering what happens when you impose the condition $\eta=0$ for the truncation. This can be easily checked, for instance using the scheme $S_{2}$. With the choice $\alpha=4 / 3$, we show from Eq. (58) that $\eta$ vanishes. This condition does not vanish $\mathcal{L}_{2}$, and the violation of the modified Ward identity holds. However, the result seems to be strongly improved in the light of the exact results obtained in this section. In particular, we show that for large melonic truncations, up to order 8 taking into account all the melons and up to order 20 for the nonbranching sector, we find an interacting fixed point with one relevant direction, whose critical exponent is always exactly equal to 2 . Once again, this result goes in the direction of our conclusions, and it seems that by taking into account the structural equations, one has comparatively greater importance even than the Ward identities concerning the convergence of the flow. However, the fixed point in question has a very bad characteristic, effective high valence couplings with very large values (of order $10^{100}$ ), meaning that the flow has moved away considerably from the Gaussian point, and once again, highlighting a very strong dependence on nonuniversal quantities at the choice of regularization.

\section{F. Closing hierarchy around the full quartic sector}

Let us briefly consider the influence of disconnected melonic pieces on the results of the previous section. As discussed above, the disconnected pieces appear as soon as $\nu \neq 1$. First of all, note that the exact relations as (96) and (98) hold, independently with the parametrization used in the phase space. Now we have the following important question which needs to be solved: What is the condition satisfied by this parametrization in agreement with the exact relation at the leading order sector?

Equation (107) holds. However, Eq. (105) has to be modified by the coupling that we called $h_{3}$ in Sec. III D:

$$
\begin{aligned}
\beta_{g, E V E}= & ((d-1)-2 \eta) \bar{g}(k)+4 \bar{g}^{2}(k) L_{3}(\eta) \\
& -8 \bar{A}_{3,0} \bar{g}^{3} L_{2}(\eta)-\bar{h}_{3} K_{2}(\eta) .
\end{aligned}
$$

The compatibility with Eq. (96) therefore requires

$$
-8 \bar{A}_{3,0} \bar{g}^{3} L_{2}-\bar{h}_{3} K_{2}=4 \bar{g}^{2} \eta \bar{A}_{3,0} .
$$

Moreover, the expression for the anomalous dimension receives a contribution for the disconnected quartic coupling (we keep the notation $g_{1}$ used in Sec. III D)

$$
\eta=-6 \bar{g} L_{2}-2 \bar{g}_{1} K_{2} .
$$

Therefore, assuming $\eta \neq 0$, we get the relation

$$
8 \bar{g}^{2} \bar{A}_{3,0}\left(2 \bar{g} L_{2}+\bar{g}_{1} K_{2}\right)-\bar{h}_{3} K_{2}=0 .
$$

We then have the explanation of the phenomenon observed in Sec. III D, i.e., the existence of the relations making the 
disconnected couplings dependent on the other couplings. From the previous section, we know that the presence of $\bar{A}_{3,0}$ introduces a spurious dependence on the initial condition. Moreover, a direct inspection shows that, adjusting $h_{3}$ to compensate the term sharing the factor $\bar{A}_{3,0}$ ultimately requires $\eta=0$, which implies that

$$
3 \bar{g} L_{2}+\bar{g}_{1} K_{2}=0,
$$

and

$$
8 \bar{g}^{3} \bar{A}_{3,0} L_{2}+\bar{h}_{3} K_{2}=0,
$$

and then discards the two last terms of (129). These two equations moreover ensure that $\dot{\bar{g}}=0 \Rightarrow \dot{\bar{g}}_{1}=\dot{\bar{h}}_{3}=0$. Therefore, the difficulties arising from the disconnected pieces seems to be solved. The strong relation between observables makes them dependent on other couplings, explaining the apparent success of ultralocal truncation. Obviously, this reasoning remains fairly qualitative, and we will endeavor to remedy the shortcomings in our future work. However, at this stage, we can ask ourselves if the convergence problems notified in Sec. III D do not come quite simply by taking into account these constraints.

\section{OPTIMIZATION CRITERIA}

At this stage we must specify our criteria for judging the quality of an approximation. Let us recall that an approximation is essentially the combination of two choices, the choice of a particular parametrization of the space of the phases given by $\Gamma_{k}$, and the choice of a regulator $r_{k}$. Usually, in FRG literature, optimization has a precise meaning. In the symmetric phase, all the loop integrals involved in the flow equations involves the effective propagator $P:=C^{-1}+r_{k}$, where $C$ denotes the bare propagator of the theory. This effective propagator has a minimum, whose position depends on the choice of $r_{k}$. More generally, as the development of the effective action takes place around a nonzero vacuum, and the minimum of $P$, we remove the risk of seeing a singularity develop around the nonzero vacuum. A regulator is then optimal when the lower bound of the free propagator $P$ is minimal. This is the sense given by Litim for optimization, and this is a very general criterion, essentially independent on the specificity of the problem that we consider. The so-called Litim regulator is optimal in this sense. This is as well the case of the regulator with $\alpha>1$ that we considered in schemes $S_{1}$ and $S_{2}$. However, in this paper, the notion of optimization is quite different and may be summarized as follows. Among a more or less large set of regulators (optimal in the Litim sense), the optimal choice(s) is such that

(i) the calculation of universal quantities such that the critical exponents are as close as possible to the exact results available, or the speed of convergence to these exact results is most important when the order of the truncation increases;

(ii) the disagreement with the set of constraints on the observables (coming for example from the symmetries of field theory) remains as small as possible, and does not increase with the order of truncation, to the orders corresponding to the effects that we hope to update; and

(iii) the computation of the universal quantities, in a scheme satisfying the two previous requirements, should not change too much under slight modifications of the regulator.

We showed in the previous section how the two first requirements work for practical calculations.

\section{DERIVATIVE EXPANSION: A FIRST LOOK}

In this section, we provide a first look about an alternative way to deal with modified Ward identities violations: introducing derivative operators on the truncation itself. We do not provide a deep investigation on this effect; the only interest is to compare this method with the other one considered in the previous section. We provide a solution at the same level of approximation, from an approximate solution solving only the first Ward identity (13). We consider a local truncation of the form

$$
\begin{aligned}
& \Gamma_{k}[M, \bar{M}]=\gamma(k)\left({ }^{*} \cdot+\infty+\infty+\infty\right) \\
& +Z(k) \propto+g(k) \sum_{i=1}^{d} \bigodot_{i}^{\infty}+\cdots,
\end{aligned}
$$

where the cross on the link of color $i$ denotes insertion of the "derivative" operator $n_{i} / k$. For instance,

$$
\stackrel{1 *}{\rightleftharpoons} \equiv \sum_{n_{1}, n_{2}, n_{3}} \frac{n_{1}}{k} \bar{M}_{\vec{n}} M_{\vec{n}} .
$$

With this definition, we have [see Eq. (13)]:

$$
\gamma(k) \equiv \frac{d}{d x_{1}} \gamma_{k}^{(2)},
$$

and therefore, from (13), $\gamma(k)$ must be related to $\bar{g}(k)$ as

$$
2 \bar{g} \overline{\mathcal{L}}_{2}=-\bar{\gamma}(k) .
$$

We introduced the notation $\overline{\mathcal{L}}_{n}$, denoting the dimensionless version of the quantity $\mathcal{L}_{n}$, discarding the $k$ and $Z$ dependence. Moreover, we defined the renormalized $\bar{\gamma}$ as

$$
\bar{\gamma}(k):=\frac{1}{Z} \gamma(k) .
$$


The flow equations for $g$ and $\gamma$ can be easily deduced from (1). Indeed, the expression for $\beta_{g}$ remains the same as computed in (68):

$$
\beta_{g}=((d-1)-2 \eta) \bar{g}+4 \bar{g}^{2} L_{3}(\eta)-\left(3 \bar{h}_{1}+2 \bar{h}_{2}\right) L_{2}(\eta),
$$

where $\eta$ is given by Eq. (122). The equation for $\gamma$ can be deduced taking the first derivative of (101) with respect to $n_{1}$. It is easy to check that the derivative of the effective loop involving $\dot{r}_{k}\left(G^{(2)}\right)^{2}$ vanishes in the large $k$ limit. Then only the derivative of the effective vertex contributes; we then get $\left(\beta_{\gamma} \equiv \dot{\bar{\gamma}}\right)$ :

$$
\beta_{\gamma}=-\eta \bar{\gamma}-2 \frac{d \bar{\pi}_{k}^{(2)}}{d x_{1}}(0,0) L_{2}(\eta)
$$

The equation for the first derivative of the effective 4-point vertex can be obtained taking the fourth order derivative of the full Ward identity (6), and vanishing external momenta. We can easily prove that

$$
\left(\left(6 h_{1}+4 h_{2}\right) \mathcal{L}_{2}-8 g^{2} \mathcal{L}_{3}\right)=-2 \frac{d}{d n} \pi_{k}^{(2)}(0,0) .
$$

Similarity from (142), we obtain

$$
\beta_{\gamma}=-\eta \bar{\gamma}-2\left(\left(3 \bar{h}_{1}+2 \bar{h}_{2}\right) \overline{\mathcal{L}}_{2}-4 \bar{g}^{2} \overline{\mathcal{L}}_{3}\right) L_{2}(\eta) .
$$

We focus on Litim regulator, and $\overline{\mathcal{L}}_{n}$ can be easily computed. Using the notation of Sec. III A, it is not difficult to check that $(\alpha=1)$ :

$$
\overline{\mathcal{L}}_{n}=d^{2} \int_{0}^{1} \frac{x^{n-1}}{\left(1+d \bar{\gamma} x^{2}\right)^{2}} \equiv \chi_{n-1,2}
$$

where we introduced $\chi_{p, q}$ defined as

$$
\chi_{p, q}:=d^{2} \int_{0}^{\alpha} \frac{x^{p}}{\left(1+d \bar{\gamma} x^{2}\right)^{q}} .
$$

For $d=3$, the integral can be easily computed using a simple integration by parts. For instance,

$$
\overline{\mathcal{L}}_{2}=d^{2} \int_{0}^{1} \frac{x}{\left(1+d \bar{\gamma} x^{2}\right)^{2}}=-\frac{d^{2}}{2 d \bar{\gamma}} \int_{0}^{1} \frac{d}{d x} \frac{1}{1+d \bar{\gamma} x^{2}},
$$

leading to

$$
\overline{\mathcal{L}}_{2}=-\frac{d^{2}}{2} \frac{1}{1+d \bar{\gamma}}
$$

We will need also to write the explicit expression for $\mathcal{L}_{3}$ :

$$
\begin{aligned}
\overline{\mathcal{L}}_{3} & =d^{2} \int_{0}^{1} \frac{x^{2}}{\left(1+d \bar{\gamma} x^{2}\right)^{2}} \\
& =-\frac{9}{6 \bar{\gamma}}\left(\left.\frac{x}{1+3 \bar{\gamma} x^{2}}\right|_{0} ^{1}-\int_{0}^{1} d x \frac{1}{1+3 \bar{\gamma} x^{2}}\right) \\
& =-\frac{9}{6 \bar{\gamma}}\left(\frac{1}{1+3 \bar{\gamma}}-\frac{1}{\sqrt{3 \bar{\gamma}}} \arctan (\sqrt{3 \bar{\gamma}})\right) .
\end{aligned}
$$

From (147) the Ward identity becomes

$$
\bar{\gamma}=d^{2} \bar{g} \frac{1}{1+d \bar{\gamma}},
$$

which can be solved as ${ }^{9}$

$$
\bar{\gamma}=-\frac{1}{6}(1-\sqrt{1+108 \bar{g}}) .
$$

Then, differentiating this relation with respect to $t=\ln (k)$, we get

$$
\beta_{\gamma}=\frac{9}{\sqrt{1+108 \bar{g}}} \beta_{g}=\frac{9}{1+6 \bar{\gamma}} \beta_{g} .
$$

Then, from explicit expressions for $\beta_{g}$ and $\beta_{\gamma}$, we deduce a relation between $3 \bar{h}_{1}+2 \bar{h}_{2}$ and $\bar{g}$ and $\bar{\gamma} ; 3 \bar{h}_{1}+2 \bar{h}_{2}=$ $h(\bar{g}, \bar{\gamma})$, with

$$
h(\bar{g}, \bar{\gamma})=(1+6 \bar{\gamma}) \frac{\eta A(\bar{\gamma})+18 \bar{g} \frac{(1-\eta)+9 \bar{g}\left(\frac{\eta}{10}+\frac{1}{2}\right)}{1+6 \bar{\gamma}}}{27\left(\frac{\eta}{4}+1\right)\left(1+\frac{1+6 \bar{\gamma}}{1+3 \bar{\gamma}}\right)},
$$

and

$$
A(\bar{\gamma}):=\bar{\gamma}-\frac{2}{3}\left(1-\frac{1+3 \bar{\gamma}}{\sqrt{3 \bar{\gamma}}} \arctan (\sqrt{3 \bar{\gamma}})\right) .
$$

We can remark that the nonbranching sector is relevant for fixed point investigations, especially for the double scaling limit. Vanishing $h_{2}$, and from the relation (149), we have

$$
3 \bar{h}_{1}=h\left(\frac{\bar{\gamma}(1+3 \bar{\gamma})}{9}, \bar{\gamma}\right)=: H(\bar{\gamma}),
$$

and the flow equations reduces to a single relation:

$$
\begin{aligned}
\beta_{\gamma}= & -\eta \bar{\gamma}+27 \frac{H(\bar{\gamma})}{1+3 \bar{\gamma}}\left(1+\frac{\eta}{4}\right) \\
& +\frac{\eta}{9}\left(1-\frac{1+3 \bar{\gamma}}{\sqrt{3 \bar{\gamma}}} \arctan (\sqrt{3 \bar{\gamma}})\right),
\end{aligned}
$$

where in this equation $\eta$ have to be expressed in term of $\bar{\gamma}$, explicitly:

\footnotetext{
${ }^{9}$ The other solution $\bar{\gamma}=-\frac{1}{6}(1+\sqrt{1+108 \bar{g}})$ does not vanish for $\bar{g} \rightarrow 0$.
} 


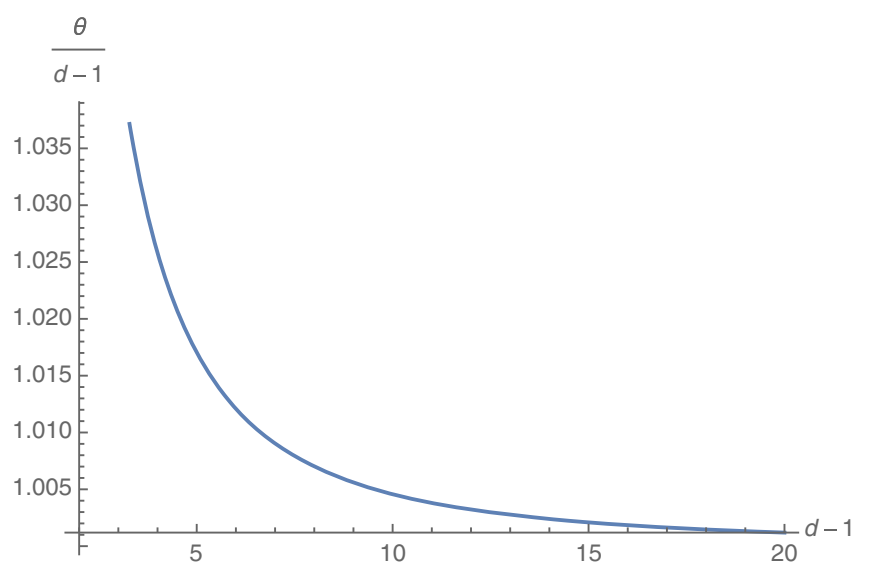

FIG. 13. Dependence of $\theta$ with the rank using the regularization scheme $S_{2}$.

$$
\eta \equiv-\frac{4(1+3 \bar{\gamma}) \bar{\gamma}}{2+(1+3 \bar{\gamma}) \bar{\gamma}}
$$

Interestingly, taking into account the first order deviation from ultralocality allows one to describe all the nonbranching sectors with a single flow equation, (155). Indeed, we expressed $h_{1}$ in terms of $\bar{g}$ and $\bar{\gamma}$, and the flow equation for $\bar{h}_{1}$ allows us to fix $u_{1}$, the octic coupling, and so on. Obviously, we discarded all the higher derivatives, and the momentum dependence of the observables with valence higher than 2 . Nevertheless, we illustrate on this simple example how the interdependence between local and nonlocal observables coming from Ward identities may have consequences on entire sectors.

We may investigate the fixed point structure of the flow equation (155). Figure 14 represents the effective $\beta$ function $\beta_{\gamma}$. Among the zeros of the $\beta$ function, only $\bar{\gamma} \approx$ -0.15 seems to be relevant. The corresponding critical exponent is $\theta \approx 1.77$ and the anomalous dimension

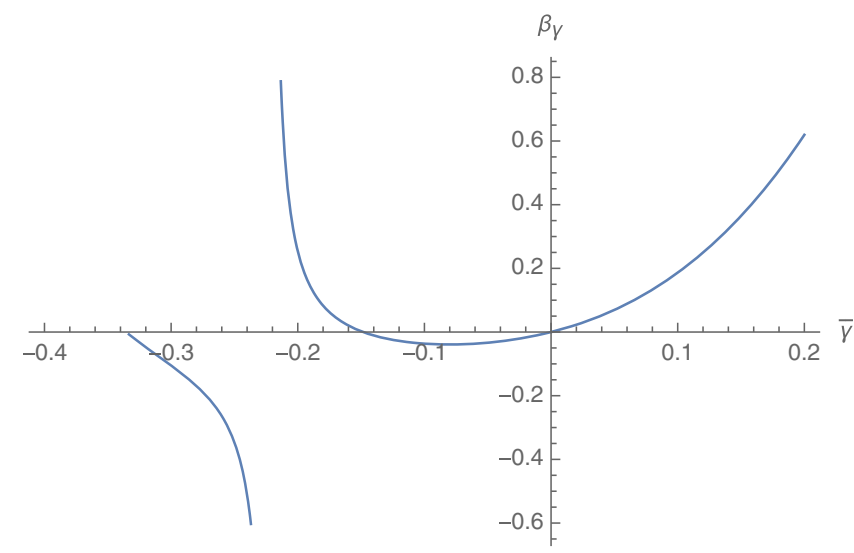

FIG. 14. Numerical plot of the function $\beta_{\gamma}$. We see that it has two zeros, for $\bar{\gamma}=0$ and $\bar{\gamma} \approx-0.15$. Moreover, it has a vanishing limit point at $\bar{\gamma} \approx-0.33$, beyond which the function becomes imaginary. $\eta \approx 0.17$. As we observed, taking into account the first Ward identity this improves strongly the result. Note that because our truncation is nonlocal, the bound $\theta_{\text {op }}=d-1$ for local truncations does not hold. The Fig. 13 provides the dependence of $\theta$ with respect to the rank of the tensor, by using the regularization scheme $S_{2}$.

In regard to the EVE, as mentioned at the beginning of Sec. IIIE, the fact that we take into account all the momentum dependence of the effective vertices allows one in principle to go beyond the dressed local potential approximation that we considered. There is in particular a very interesting aspect or the melonic EVE: In the melonic sector, all the relations between effective vertex functions due to the EVE are compatible with Ward identities, meaning that no additional assumptions are required to deal with them. The same thing has been observed for a subleading sector in [79], and it is tempting to conjecture that it must be a general property of EVE, sector by sector in the $1 / N$ expansion. This property can be easily checked for the melonic sector. From Sec. III E, we know that the melonic self-energy per color $\sigma_{k}(n)$ must satisfy the closed equation (100). Taking the first derivative with respect to the external momenta $n$, we get (note that we set $\nu=d$ for this section):

$$
\frac{d \sigma_{k}}{d n}=2 g \sum_{\vec{n}} \delta_{n_{1} n} \frac{-\frac{d \sigma_{k}}{d n}+\frac{d r_{k}}{d n}}{\left(1-\sum_{i=1}^{d} \sigma\left(n_{i}\right)+r_{k}(\vec{n})\right)^{2}} .
$$

From the definitions of $\mathcal{L}_{p}$ [see Eq. (14)], $\mathcal{A}_{m, n}$ [see Eq. (97)], and $\gamma \equiv-d \sigma_{k} / d n(n=0)$, we get, setting $n=0$,

$$
-\gamma=2 g \mathcal{L}_{2}+2 g \gamma \mathcal{A}_{2,0}
$$

or simply

$$
\gamma=-2\left(\frac{g}{1+2 g \mathcal{A}_{2,0}}\right) \mathcal{L}_{2} .
$$

Then, from Eq. (96), the bracket term is nothing but $\pi_{k}^{(2)}(0,0)$, and the previous equation reduces to the Ward identity (13). The same compatibility can be checked for higher order Ward identities. For instance, restricting to the nonbranching sector, we deduce from (142)

$$
\left(\pi_{k}^{(3)}(0,0,0) \mathcal{L}_{2}-8\left(\pi_{k}^{(2)}(0,0)\right)^{2} \mathcal{L}_{3}\right)=-2 \frac{d}{d n} \pi_{k}^{(2)}(0,0)
$$

in the same way as we derive in Eq. (13). From Eq. (96), the derivative can be easily computed, leading to

$$
\pi_{k}^{(3)}(0,0,0) \mathcal{L}_{2}=4\left(\pi_{k}^{(2)}(0,0)\right)^{2}\left[\frac{d A_{2,0}}{d n}+2 \mathcal{L}_{3}\right] \text {. }
$$

Moreover, as for Eq. (157), it is easy to compute the derivative of $A_{2, n}$. Using once again the fact that, in the melonic sector the free energy decomposes as $\Sigma_{k}(\vec{n})=$ $\sum_{i} \sigma_{k}\left(n_{i}\right)$, we get straightforwardly 


$$
\frac{d A_{2,0}}{d n}=-2 \sum_{\vec{n}} \delta_{n_{1} 0} \frac{-\frac{d \sigma_{k}}{d n}+\frac{d r_{k}}{d n}}{\left(1-\sum_{i=1}^{d} \sigma\left(n_{i}\right)+r_{k}(\vec{n})\right)^{3}},
$$

and therefore

$$
\frac{d A_{2,0}}{d n}-2 \mathcal{L}_{3}=-2 \gamma A_{3,0}
$$

Finally, from Eq. (96) and Ward identity (13), we get

$$
\pi_{k}^{(3)}(0,0,0) \mathcal{L}_{2}=2^{4}\left(\pi_{k}^{(2)}(0,0)\right)^{3} \gamma A_{3,0} \mathcal{L}_{2},
$$

from which we recognize the expression of $\pi_{k}^{(3)}(0,0,0)$ given by the EVE, Eq. (98). Note that the proof seems to be very dependent on the fact that Eq. (93) holds, especially for 2-point functions. Such a condition, however, could be lost for subleading orders [79], which may require additional conditions regarding Ward identities.

\section{CONCLUSION}

In this paper, we have essentially focused on the compatibility between local truncations and exact relations between observables at the large $N$ limit. These relations, moreover, have different natures. The modified Ward identities come from the internal symmetry group used to define the allowed interactions, and the structure equations are nothing but ordinary Schwinger-Dyson equations in the melonic sector. With this respect, these relations may be understood in two different manners. On the one hand, Schwinger-Dyson equations are the consequence of the formal Lebesgue measure involved in the path integral definition of the partition function [96]. On the other hand, the structure equations may be derived directly in the large $N$ limit as a consequence of the recursive definition of melons [84]. To put it in a nutshell, we showed the following:

(i) Accommodating these constraints, we improved the rate of convergence toward a given limit in a given sector, this limit depending on the sector that we consider.

(ii) The flow seems to be more sensitive to the structural constraints, arising from the Schwinger-Dyson equations, than to the symmetry constraint given by the modified Ward identities.

One expects that the second point is a consequence of the fact that generating the flow requires a symmetry breaking, modifying the Ward identities, while the structure equations remain formally unchanged. Despite the existence of a fixed point having a single relevant direction, and reminiscent to the critical scenario of the double scaling, two difficulties appeared in the light of this study. The first is that a considerable number of fixed points generally accompany this (fixed points which generally have more than one lifting direction, and can persist in high truncations). This is not clearly understood because, although it is tempting to interpret these fixed points as a possible multicritical fixed point, it corresponds to different limits beyond double scaling. It has been shown that an analysis taking into account the close relations between observables coming from the structural equations discard these residual fixed points. The effect of Ward identities moreover seems less crucial. Indeed, it is easy to check that taking into account the locality constraint coming from the structure equations, $\eta=0$, provides a fixed point with one relevant direction having exactly the limit value $\theta=2$ for any truncations (up to order 20). In contrast, to accommodate Ward identities, at least in the first order of derivative expansion, implies slow convergence phenomena. These conclusions will be straightforwardly extended to the real models based on the internal group $\mathrm{O}(N)$, which probably remains in agreement with the conclusions of the recent work [80] and which remains a subject of forthcoming work.

Thus, we then expect that this work provides a serious way of reflection and investigation on the methods used to compute the critical behavior of random tensor models. We have pointed out the crucial role played by exact functional relations, but we focused only on the leading order in the $1 / N$ expansion. The EVE method for sectors beyond melons quickly becomes intractable, as showed in [79]. A promising way, outlined in this paper, could be to "dress" a few complete sectors with truncations, taking care for each new magnitude explored for the coupling constants, the violation of the different exact relation is available. This strategy should be explored in an upcoming article and will help to increase confidence in the validity of the results made in the deep regions of the phase space. Finally, deeper investigations about integrability and regularity of the resulting RG flow has yet to be done for RMMs and RTMs, for the considered approximations. These aspects will be considered in future work.

\section{ACKNOWLEDGMENTS}

V.L. gives special thanks to Laetitia Mercey for her constant support during the writing of this article.

\section{APPENDIX: USEFUL INTEGRALS}

Let us consider integrals of the form

$$
J=\int_{0}^{\infty} d x_{1} d x_{2} \theta\left(\alpha-x_{1}-x_{2}\right) f\left(x_{1}+x_{2}\right)
$$

Firstly, we set $x_{i}=y_{i}^{2}$, leading to

$$
J=4 \int_{0}^{\infty} d y_{1} d y_{2} y_{1} y_{2} \theta\left(\alpha-r^{2}\right) f\left(r^{2}\right),
$$

where $r^{2}:=y_{1}^{2}+y_{2}^{2}$. Then, we use polar coordinates, $d y_{1} d y_{2}=r d r d \varphi, y_{1}=r \cos (\varphi), y_{2}=r \sin (\varphi)$, leading to 


$$
J=\left(\int_{0}^{\frac{\pi}{2}} d \varphi 2 \sin (\varphi) \cos (\varphi)\right) \int_{0}^{\alpha} d r^{2} r^{2} \theta\left(\alpha-r^{2}\right) f\left(r^{2}\right),
$$

where we restricted our analysis to the angular domain in the region where both $y_{1}$ and $y_{2}$ are positives. Finally,

$$
J=\int_{0}^{\alpha} d x x f(x)
$$

In the same way, we consider the three-dimensional integral:

$K=\int d x_{1} d x_{2} d x_{3} f\left(x_{1}+x_{2}+x_{3}\right) \theta\left(\alpha-x_{1}-x_{2}-x_{3}\right)$.

We introduce $y_{i}$ defined as $x_{i}=y_{i}^{2}$, and $r^{2}=\sum_{i} y_{i}^{2}$, so that

$$
K=8 \int d^{3} y\left(y_{1} y_{2} y_{3}\right) f\left(r^{2}\right) \theta\left(\alpha-r^{2}\right) .
$$

Then, introducing the polar coordinates,

$$
\begin{aligned}
& y_{1}=r \cos (\vartheta), \\
& y_{2}=r \cos (\varphi) \sin (\vartheta), \\
& y_{3}=r \sin (\varphi) \sin (\vartheta),
\end{aligned}
$$

the integral $K$ becomes

$$
\begin{aligned}
K= & 8 \int_{0}^{\frac{\pi}{2}} d \vartheta \int_{0}^{\frac{\pi}{2}} d \varphi \cos (\vartheta) \sin ^{3}(\vartheta) \cos (\varphi) \sin (\varphi) \\
& \times \int_{0}^{\sqrt{\alpha}} d r r^{5} f\left(r^{2}\right) .
\end{aligned}
$$

The angular integrals can be easily computed, and we find

$8 \int_{0}^{\frac{\pi}{2}} d \vartheta \int_{0}^{\frac{\pi}{2}} d \varphi \cos (\vartheta) \sin ^{3}(\vartheta) \cos (\varphi) \sin (\varphi)=1$,

and finally, introducing $x=r^{2}$,

$$
K=\frac{1}{2} \int_{0}^{\alpha} x^{2} f(x) d x
$$

[1] H. Ooguri and N. Sasakura, Discrete and continuum approaches to three-dimensional quantum gravity, Mod. Phys. Lett. A 06, 3591 (1991).

[2] N. Sasakura, Tensor model for gravity and orientability of manifold, Mod. Phys. Lett. A 06, 2613 (1991).

[3] H. Ooguri, Schwinger-Dyson equation in three-dimensional simplicial quantum gravity, Prog. Theor. Phys. 89, 1 (1993).

[4] D. V. Boulatov, A model of three-dimensional lattice gravity, Mod. Phys. Lett. A 07, 1629 (1992).

[5] N. Godfrey and M. Gross, Simplicial quantum gravity in more than two dimensions, Phys. Rev. D 43, R1749 (1991).

[6] M. Gross, Tensor models and simplicial quantum gravity in 2-D, Nucl. Phys. B, Proc. Suppl. 25, 144 (1992).

[7] E. Brezin and J. Zinn-Justin, Renormalization group approach to matrix models, Phys. Lett. B 288, 54 (1992).

[8] P. Di Francesco, P. H. Ginsparg, and J. Zinn-Justin, 2-D Gravity and random matrices, Phys. Rep. 254, 1 (1995).

[9] S. Higuchi, C. Itoi, and N. Sakai, Renormalization group approach to matrix models and vector models, Prog. Theor. Phys. Suppl. 114, 53 (1993).

[10] J. Zinn-Justin, Random vector and matrix and vector theories: A renormalization group approach, J. Stat. Phys. 157, 990 (2014).

[11] J. Ambjorn, L. Chekhov, C. F. Kristjansen, and Y. Makeenko, Matrix model calculations beyond the spherical limit, Nucl. Phys. B404, 127 (1993); Erratum, Nucl. Phys. B449, 681 (1995).
[12] J. Ambjorn, J. Jurkiewicz, and C. F. Kristjansen, Quantum gravity, dynamical triangulations and higher derivative regularization, Nucl. Phys. B393, 601 (1993).

[13] R. Gurau and J. P. Ryan, Colored tensor models: A review, SIGMA 8, 020 (2012).

[14] R. Gurau, Invitation to random tensors, SIGMA 12, 094 (2016).

[15] V. Rivasseau, The tensor theory space, Fortschr. Phys. 62, 835 (2014).

[16] R. Gurau, Colored group field theory, Commun. Math. Phys. 304, 69 (2011).

[17] V. Rivasseau, Random tensors and quantum gravity, SIGMA 12, 069 (2016).

[18] R. Gurau, The complete $1 / N$ expansion of colored tensor models in arbitrary dimension, Ann. Inst. Henri Poincaré 13, 399 (2012).

[19] R. Gurau, The $1 / N$ expansion of colored tensor models, Ann. Inst. Henri Poincaré 12, 829 (2011).

[20] R. Gurau, The $1 / N$ expansion of tensor models beyond perturbation theory, Commun. Math. Phys. 330, 973 (2014).

[21] R. Gurau and J. P. Ryan, Melons are branched polymers, Ann. Inst. Henri Poincaré 15, 2085 (2014).

[22] V. Bonzom, R. Gurau, and V. Rivasseau, The Ising model on random lattices in arbitrary dimensions, Phys. Lett. B 711, 88 (2012).

[23] V. Bonzom, R. Gurau, A. Riello, and V. Rivasseau, Critical behavior of colored tensor models in the large $N$ limit, Nucl. Phys. B853, 174 (2011). 
[24] V. Bonzom, R. Gurau, and V. Rivasseau, Random tensor models in the large $N$ limit: Uncoloring the colored tensor models, Phys. Rev. D 85, 084037 (2012).

[25] V. Bonzom, Large $N$ limits in tensor models: Towards more universality classes of colored triangulations in dimension $d \geq 2$, SIGMA 12, 073 (2016).

[26] V. Bonzom, Tensor models with generalized melonic interactions, arXiv:1905.01903.

[27] V. Bonzom, V. Nador, and A. Tanasa, Diagrammatics of the quartic $O(N)^{3}$-invariant Sachdev-Ye-Kitaev-like tensor model, J. Math. Phys. (N.Y.) 60, 072302 (2019).

[28] D. Benedetti, R. Gurau, S. Harribey, and K. Suzuki, Hints of unitarity at large $N$ in the $O(N)^{3}$ tensor field theory, J. High Energy Phys. 02 (2020) 072.

[29] D. Benedetti, S. Carrozza, R. Toriumi, and G. Valette, Multiple scaling limits of $\mathrm{U}(N)^{2} \times \mathrm{O}(D)$ multimatrix models, arXiv:2003.02100.

[30] D. Benedetti, R. Gurau, and K. Suzuki, Conformal symmetry and composite operators in the $O(N)^{3}$ tensor field theory, J. High Energy Phys. 06 (2020) 113.

[31] D. Benedetti and I. Costa, $S O(3)$-invariant phase of the $O(N)^{3}$ tensor model, Phys. Rev. D 101, 086021 (2020).

[32] R. Gurau, The complete $1 / N$ expansion of a SYK-like tensor model, Nucl. Phys. B916, 386 (2017).

[33] R. Gurau, Quenched equals annealed at leading order in the colored SYK model, Europhys. Lett. 119, 30003 (2017).

[34] R. Gurau, The $\imath \epsilon$ prescription in the SYK model, J. Phys. Commun. 2, 015003 (2018).

[35] D. Benedetti, S. Carrozza, R. Gurau, and A. Sfondrini, Tensorial Gross-Neveu models, J. High Energy Phys. 01 (2018) 003.

[36] P. Narayan and J. Yoon, SYK-like tensor models on the lattice, J. High Energy Phys. 08 (2017) 083.

[37] V. Bonzom, L. Lionni, and A. Tanasa, Diagrammatics of a colored SYK model and of an SYK-like tensor model, leading and next-to-leading orders, J. Math. Phys. (N.Y.) 58, 052301 (2017).

[38] I. R. Klebanov and G. Tarnopolsky, Uncolored random tensors, melon diagrams, and the Sachdev-Ye-Kitaev models, Phys. Rev. D 95, 046004 (2017).

[39] J. Kim, I. R. Klebanov, G. Tarnopolsky, and W. Zhao, Symmetry Breaking in Coupled SYK or Tensor Models, Phys. Rev. X 9, 021043 (2019).

[40] J. Kim, Large $N$ tensor and SYK models, arXiv:1811 .04330 .

[41] N. Delporte and V. Rivasseau, Perturbative quantum field theory on random trees, arXiv:1905.12783.

[42] D. Oriti, Group field theory and simplicial quantum gravity, Classical Quantum Gravity 27, 145017 (2010).

[43] C. Rovelli, Loop quantum gravity, Living Rev. Relativity 1, 1 (1998).

[44] C. Rovelli and P. Upadhya, Loop quantum gravity and quanta of space: A Primer, arXiv:gr-qc/9806079.

[45] S. Carrozza, D. Oriti, and V. Rivasseau, Renormalization of a SU(2) tensorial group field theory in three dimensions, Commun. Math. Phys. 330, 581 (2014).

[46] J. Ben Geloun, Renormalizable models in rank $d \geq 2$ tensorial group field theory, Commun. Math. Phys. 332, 117 (2014).
[47] V. Lahoche and D. Oriti, Renormalization of a tensorial field theory on the homogeneous space $\mathrm{SU}(2) / \mathrm{U}(1)$, J. Phys. A 50, 025201 (2017).

[48] V. Lahoche, D. Oriti, and V. Rivasseau, Renormalization of an Abelian tensor group field theory: Solution at leading order, J. High Energy Phys. 04 (2015) 095.

[49] J. Ben Geloun and E. R. Livine, Some classes of renormalizable tensor models, J. Math. Phys. (N.Y.) 54, 082303 (2013).

[50] D. O. Samary and F. Vignes-Tourneret, Just renormalizable TGFT's on $U(1)^{d}$ with gauge invariance, Commun. Math. Phys. 329, 545 (2014).

[51] J. Ben Geloun and D. O. Samary, 3D tensor field theory: Renormalization and one-loop $\beta$ functions, Ann. Inst. Henri Poincaré 14, 1599 (2013).

[52] J. Ben Geloun and V. Rivasseau, A renormalizable 4-dimensional tensor field theory, Commun. Math. Phys. 318, 69 (2013).

[53] S. Carrozza, Discrete renormalization group for $\mathrm{SU}(2)$ tensorial group field theory, Ann. Inst. Henri Poincaré 2, 49 (2015).

[54] J. B. Geloun, R. Martini, and D. Oriti, Functional renormalzsation group analysis of tensorial group field theories on $\mathbb{R}^{d}$, Phys. Rev. D 94, 024017 (2016).

[55] J. B. Geloun, R. Martini, and D. Oriti, Functional renormalization group analysis of a tensorial group field theory on $\mathbb{R}^{3}$, Europhys. Lett. 112, 31001 (2015).

[56] D. Benedetti and V. Lahoche, Functional renormalization group approach for tensorial group field theory: A rank-6 model with closure constraint, Classical Quantum Gravity 33, 095003 (2016).

[57] D. Benedetti, J. Ben Geloun, and D. Oriti, Functional renormalisation group approach for tensorial group field theory: A rank-3 model, J. High Energy Phys. 03 (2015) 084.

[58] D. Benedetti, R. Gurau, and S. Harribey, Line of fixed points in a bosonic tensor model, J. High Energy Phys. 06 (2019) 053.

[59] J. Ben Geloun, T. A. Koslowski, D. Oriti, and A. D. Pereira, Functional renormalization group analysis of rank 3 tensorial group field theory: The full quartic invariant truncation, Phys. Rev. D 97, 126018 (2018).

[60] S. Carrozza and V. Lahoche, Asymptotic safety in threedimensional SU(2) group field theory: Evidence in the local potential approximation, Classical Quantum Gravity 34, 115004 (2017).

[61] V. Lahoche and D. O. Samary, Functional renormalization group for the $\mathrm{U}(1)-\mathrm{T}_{5}^{6}$ tensorial group field theory with closure constraint, Phys. Rev. D 95, 045013 (2017).

[62] S. Carrozza, V. Lahoche, and D. Oriti, Renormalizable group field theory beyond melonic diagrams: An example in rank four, Phys. Rev. D 96, 066007 (2017).

[63] S. Higuchi, C. Itoi, S. Nishigaki, and N. Sakai, Renormalization group flow in one and two matrix models, Nucl. Phys. B434, 283 (1995); Erratum, Nucl. Phys. B441, 405 (1995).

[64] L. Canet, B. Delamotte, D. Mouhanna, and J. Vidal, Nonperturbative renormalization group approach to the Ising model: A Derivative expansion at order partial**4, Phys. Rev. B 68, 064421 (2003). 
[65] J. Alfaro and P. H. Damgaard, The $D=1$ matrix model and the renormalization group, Phys. Lett. B 289, 342 (1992).

[66] D. Stanford and E. Witten, JT gravity and the ensembles of random matrix theory, arXiv:1907.03363.

[67] R. Gurau, Notes on tensor models and tensor field theories, arXiv:1907.03531.

[68] J. Ambjorn, J. Jurkiewicz, S. Varsted, A. Irback, and B. Petersson, Critical properties of the dynamical random surface with extrinsic curvature, Phys. Lett. B 275, 295 (1992).

[69] P. H. Ginsparg and G. W. Moore, Lectures on 2-D gravity and 2-D string theory, arXiv:hep-th/9304011.

[70] K. Itoh, Gauge symmetry and the functional renormalization group, Int. J. Mod. Phys. A 32, 1747011 (2017).

[71] H. B. Gao, On renormalization group flow in matrix model, arXiv:hep-th/9209089.

[72] C. Ayala, Renormalization group approach to matrix models in two-dimensional quantum gravity, Phys. Lett. B 311, 55 (1993).

[73] A. Sfondrini and T. A. Koslowski, Functional renormalization of noncommutative scalar field theory, Int. J. Mod. Phys. A 26, 4009 (2011).

[74] A. Eichhorn and T. Koslowski, Continuum limit in matrix models for quantum gravity from the functional renormalization group, Phys. Rev. D 88, 084016 (2013).

[75] A. Eichhorn and T. Koslowski, Towards phase transitions between discrete and continuum quantum spacetime from the renormalization group, Phys. Rev. D 90, 104039 (2014).

[76] C. Wetterich, The average action for scalar fields near phase transitions, Z. Phys. C 57, 451 (1993).

[77] C. Wetterich, Exact evolution equation for the effective potential, Phys. Lett. B 301, 90 (1993).

[78] V. Lahoche and D. O. Samary, Ward identity violation for melonic $T^{4}$-truncation, Nucl. Phys. B940, 190 (2019).

[79] V. Lahoche and D. O. Samary, Nonperturbative renormalization group beyond the melonic sector: The effective vertex expansion method for group fields theories, Phys. Rev. D 98, 126010 (2018).

[80] V. Lahoche and D. O. Samary, Revisited functional renormalization group approach for random matrices in the large- $N$ limit, Phys. Rev. D 101, 106015 (2020).

[81] V. Lahoche and D. O. Samary, Pedagogical comments about nonperturbative Ward-constrained melonic renormalization group flow, Phys. Rev. D 101, 024001 (2020).

[82] V. Lahoche and D. O. Samary, Ward-constrained melonic renormalization group flow, Phys. Lett. B 802, 135173 (2020).

[83] V. Lahoche and D. O. Samary, Progress in the solving nonperturbative renormalization group for tensorial group field theory, Universe 5, 86 (2019).

[84] V. Lahoche and D. O. Samary, Unitary symmetry constraints on tensorial group field theory renormalization group flow, Classical Quantum Gravity 35, 195006 (2018).

[85] V. Lahoche, D. O. Samary, and A. D. Pereira, Renormalization group flow of coupled tensorial group field theories: Towards the Ising model on random lattices, Phys. Rev. D 101, 064014 (2020).
[86] V. Lahoche and D. O. Samary, Ward-constrained melonic renormalization group flow for the rank-four $\phi^{6}$ tensorial group field theory, Phys. Rev. D 100, 086009 (2019).

[87] C. Wetterich, Gauge invariant flow equation, Nucl. Phys. B931, 262 (2018).

[88] F. Freire, D. F. Litim, and J. M. Pawlowski, Gauge invariance, background fields and modified Ward identities, Int. J. Mod. Phys. A 16, 2035 (2001).

[89] C. Wetterich, Gauge-invariant fields and flow equations for Yang-Mills theories, Nucl. Phys. B934, 265 (2018).

[90] M. Safari, Splitting Ward identity, Eur. Phys. J. C 76, 201 (2016).

[91] D. F. Litim, Optimization of the exact renormalization group, Phys. Lett. B 486, 92 (2000).

[92] D. F. Litim, Derivative expansion and renormalization group flows, J. High Energy Phys. 11 (2001) 059.

[93] L. Canet, B. Delamotte, D. Mouhanna, and J. Vidal, Optimization of the derivative expansion in the nonperturbative renormalization group, Phys. Rev. D 67, 065004 (2003).

[94] B. Delamotte, An introduction to the nonperturbative renormalization group, Lect. Notes Phys. 852, 49 (2012).

[95] J. Ben Geloun, Ward-Takahashi identities for the colored Boulatov model, J. Phys. A 44, 415402 (2011).

[96] D. O. Samary, Closed equations of the two-point functions for tensorial group field theory, Classical Quantum Gravity 31, 185005 (2014).

[97] C. I. Prez-Snchez, The full Ward-Takahashi identity for colored tensor models, Commun. Math. Phys. 358, 589 (2018).

[98] T. R. Morris, The exact renormalization group and approximate solutions, Int. J. Mod. Phys. A 09, 2411 (1994).

[99] T. R. Morris, Equivalence of local potential approximations, J. High Energy Phys. 07 (2005) 027.

[100] T. R. Morris and J. F. Tighe, Convergence of derivative expansions in scalar field theory, Int. J. Mod. Phys. A 16, 2095 (2001).

[101] A. Eichhorn, T. Koslowski, J. Lumma, and A. D. Pereira, Towards background independent quantum gravity with tensor models, Classical Quantum Gravity 36, 155007 (2019).

[102] V. Bonzom, R. Gurau, J. P. Ryan, and A. Tanasa, The double scaling limit of random tensor models, J. High Energy Phys. 09 (2014) 051.

[103] R. Gurau, A. Tanasa, and D. R. Youmans, The double scaling limit of the multiorientable tensor model, Europhys. Lett. 111, 21002 (2015).

[104] A. Eichhorn and T. Koslowski, Flowing to the continuum in discrete tensor models for quantum gravity, Ann. Inst. Henri Poincaré 5, 173 (2018).

[105] T. Delepouve, R. Gurau, and V. Rivasseau, Universality and Borel summability of arbitrary quartic tensor models, Ann. Inst. Henri Poincaré 52, 821 (2016).

[106] D. O. Samary, C. I. Prez-Snchez, F. Vignes-Tourneret, and R. Wulkenhaar, Correlation functions of a just renormalizable tensorial group field theory: The melonic approximation, Classical Quantum Gravity 32, 175012 (2015). 